3 5

11 Kingdom.

12 dDepartment of Academic Surgery, Royal Marsden Hospital, London SW3 6JJ, United Kingdom.

13 eThe London Clinic, London W1G 6JA, United Kingdom.

14

\section{Analysis of the immunomodulatory properties of two heat-killed mycobacterial preparations in a human whole blood model}

Samer Bazzi ${ }^{a, b}$, Helmout Modjtahedi a, Satvinder Mudan ${ }^{c, d}$, Charles Akle ${ }^{e}$, Georges M. Bahr ${ }^{b, *}$

aSchool of Life Sciences, Faculty of Science, Engineering and Computing, Kingston University, Kingston upon Thames, Surrey KT1 2EE, United Kingdom.

${ }^{\text {b} F a c u l t y ~ o f ~ M e d i c i n e ~ a n d ~ M e d i c a l ~ S c i e n c e s, ~ U n i v e r s i t y ~ o f ~ B a l a m a n d, ~} 33$ Amioun, Al Kurah, Lebanon.

'Division of Clinical Sciences, St George's, University of London, London SW170RE, United

\section{Email addresses}

Samer Bazzi: k1170034@kingston.ac.uk

Helmout Modjtahedi: H.Modjtahedi@kingston.ac.uk.com

Satvinder Mudan: mudans@aol.com

Charles Akle: cakle@me.com

Georges Bahr: georges.bahr@balamand.edu.lb

\section{${ }^{\star}$ Corresponding author}

Georges M. Bahr, Faculty of Medicine and Medical Sciences, University of Balamand, PO BOX:

33, Amioun, Al Kurah, Lebanon. Tel: 009613370780; Fax: 009616931956.

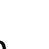

0




\section{Abstract}

The significant role played by mycobacteria in modulating immune responses through enhancing the crosstalk between innate and adaptive immunity has been highlighted in several studies. Owing to their unique antigenic profile, heat killed (HK) preparations of rapid-growing mycobacteria, currently undergoing clinical development, have been assessed as adjuvant therapy in various diseases. The purpose of this study is to investigate the regulation of leukocyte surface receptors, in whole blood from healthy donors, following in vitro stimulation with HK Mycobacterium vaccae (M. vaccae) or M. obuense. We have demonstrated the ability of both mycobacterial preparations to target monocytes and neutrophils and to regulate the surface expression of selected adhesion receptors, antigen-presenting and costimulatory receptors, pattern recognition receptors, complement and Fc receptors, as well as cytokine/chemokine receptors. Toll-like receptors (TLRs) 1 and 2 were also shown to be involved in mediating the $M$. obuense-induced upregulation of selected surface receptors on monocytes. Whole blood stimulation with $M$. vaccae or $M$. obuense resulted in a significant increase in the secretion of a specific set of cytokines and chemokines. Both mycobacterial preparations induced strong antigen-specific proliferative responses in peripheral blood mononuclear cells. Collectively, our data shows that $M$. vaccae and $M$. obuense have the potential to act as potent immunomodulators. Future research based on these findings may reveal novel immune pathways induced by these preparations with potential implication for their use in diverse immunotherapeutic approaches.

\section{2}

Keywords: Mycobacteria; immunomodulation; human leukocytes; CD antigens

Abbreviations: APC: antigen presenting cell, BCG: bacillus calmette-guerin, CCR: CC chemokine receptor, cpm: counts per minute, CR: complement receptor; CXCL: chemokine CX-C motif ligand, CXCR: chemokine C-X-C motif receptor, DC: dendritic cell, ECMR: extracellular matrix receptor, FSC: forward scatter, GM-CSFR: granulocyte-macrophage colony-stimulating factor receptor, GP: glycoprotein, HK: heat killed, ICAM: intercellular adhesion molecule, LCA: leukocyte common antigen, LFA: lymphocyte function associated antigen, LPSR: lipopolysaccharide receptor, MFI: mean fluorescence intensity, MHC: major histocompatibility complex, MIP: macrophage inflammatory protein, MMR: macrophage mannose receptor, PBMCs: peripheral blood mononuclear cells, PECAM: platelet endothelial cell adhesion molecule, RANTES: regulated upon activation normal $\mathrm{T}$ cell expressed and secreted, SSC: side scatter, SI: stimulation index, TB: tuberculosis, TLR: toll-like receptor, TNFRSF: tumor necrosis factor receptor superfamily member. 


\section{Introduction}

2 Mycobacteria, due to their diverse and complex cell wall structures, play a significant role in 3 modulating immune responses (Rook, et al. 2004; Stanford, et al. 2009) For several years, 4 Bacillus Calmette-Guerin (BCG), a live attenuated derivative of the slow-growing species 5 Mycobacterium bovis ( $M$. bovis), has been used as a prophylactic vaccine against tuberculosis 6 (TB); though, with variable efficacy (Romano and Huygen 2012). Moreover, BCG has proven to 7 be successful in the treatment of early non-invasive bladder cancer (Kawai, et al. 2013). The 8 investigation of inactivated rapid-growing mycobacteria to be employed as immunomodulators has led to the selection of $M$. vaccae which was later evaluated as an immunomodulating therapeutic agent in various diseases including TB (Dlugovitzky, et al. 2006; Johnson, et al. 2000; Yang, et al. 2011), leprosy (Abbot, et al. 2002; Truoc, et al. 2001), psoriasis (Lehrer, et al. 1998), dermatitis (Arkwright and David 2001), asthma (Camporota, et al. 2003) and a range of cancers (Cananzi, et al. 2013; Eaton, et al. 2002; O'Brien, et al. 2004; Patel, et al. 2008; Stanford, et al. 2008). M. vaccae was also found to be an effective vaccine that could confer a significant level of protection against TB among HIV-infected individuals who had received BCG vaccination during childhood (von Reyn, et al. 2010). In all these studies, M. vaccae was used in the form of a heat-killed (HK) preparation that retained its antigenic properties. In spite of the growing interest in the therapeutic implications of HK mycobacterial preparations, the nature of the host immune receptors regulated by these mycobacterial preparations has not been fully defined. Moreover, the exact mechanism by which HK mycobacterial preparations exert their immunomodulatory effects in humans is not fully understood. Nevertheless, in experimental models, immunization with $\mathrm{HK} M$. vaccae was reported to generate $\mathrm{CD}^{+}{ }^{+}$T cells against $M$. tuberculosis-infected macrophages (Skinner, et al. 1997) and to downregulate T helper type 2 (Th2) responses in murine models of allergic pulmonary inflammation via the induction of regulatory $T$ cells (Zuany-Amorim, et al. 2002) and the priming of dendritic cells (DCs) consistent with a regulatory profile (Adams, et al. 2004). In vitro studies with human monocyte derived DCs have confirmed the ability of $M$. vaccae to dampen Th2 responses, via a mechanism dependent on DCs (Le Bert, et al. 2011). Furthermore, M. vaccae and M. obuense have been shown to promote the anti-tumor activity of human yठT-cells via cytokine release from type-1 myeloid DCs (Fowler, et al. 2012). M. obuense has gained attention over the past few years as a potential immunotherapeutic agent against cancer and, in a phase 1 study in melanoma patients, it was shown to be safe and well tolerated (Stebbing, et al. 2012). More recently, in a phase 2 study, the use of $M$. obuense (NCTC13365) as adjunctive immunotherapy 
for advanced pancreatic cancer resulted in clinically meaningful increases in overall survival as well as in progression-free survival of patients (Dalgleish, A.G. and The IMAGE I Trial Investigators 2015). Currently, M. obuense (NCTC13365) is being investigated in a phase 2 study involving patients with melanoma (NCT01559818). The interactions between different immune cells and between immune cells and soluble factors are mediated by a number of cell surface receptors involved in cellular adhesion, antigen presentation and co-stimulation, cytokine-mediated signaling, and cellular activation (Barclay, et al. 1997). Therefore, variations in the expression of these receptors would affect the immune cell function thus leading to the modulation of immune responses. Immune responses from healthy volunteers, assessed by the profile of released cytokines, have been extensively analyzed using short-term incubation of human whole blood with a large spectrum of microbial immunostimulants, immunomodulators, or specific antigens (Darcissac, et al. 1996a; Darcissac, et al. 1996b; Duffy, et al. 2014; Kassa, et al. 2012; Reichenbach, et al. 2006). The immunomodulatory properties of HK M. vaccae or M. obuense in the human whole blood model have not been previously evaluated. In this study, both HK mycobacterial preparations were assessed for their ability to modulate in vitro the expression of immunologically relevant leukocyte surface receptors and to alter cytokine and chemokine secretion in human whole blood. We have also addressed the role of toll-like receptors (TLRs) in mediating the immunomodulatory effects of $M$. obuense, and evaluated the ability of $M$. vaccae and $M$. obuense to induce lymphoproliferative responses in human peripheral blood mononuclear cells (PBMCs) from healthy volunteers.

\section{Materials and Methods}

\section{Antibodies}

Mouse monoclonal anti-human antibodies purchased from BD Biosciences (San Jose, CA, USA) included: FITC-conjugated CD2 (clone RPA-2.10), CD11a (clone HI111), CD35 (clone E11), CD40 (clone 5C3), CD44 (clone L178), CD45 (clone 2D1), CD50 (clone TU41), CD64 (clone 10.1), CD80 (clone L307.4), CD95 (clone DX2), CD195 (clone 2D7/CCR5), HLADP,DQ,DR (clone Tu39); PEconjugated CD11b (clone ICRF44), (clone WM59), CD36 (clone CB38), CD58 (clone 1C3), CD89 (clone A59), CD102 (clone CBR- 1C2/2), CD114 (clone LMM741), CD116 (clone hGMCSFR-M1), CD119 (clone GIR-208), CD122 (clone TU27), CD127 (clone HIL- 7R-M21), CD132 (clone AG184), CD137L (clone C65-485), CD206 (clone 19.2), PerCP-conjugated CD14 (clone M5E2), HLA-DR (clone G46-6); PE-Cy7-conjugated CD16 (clone 3G8); APC-conjugated CD5 (clone UCHT2), CD11c (clone B-ly6), CD18 (clone 6.7), CD21 (clone B-ly4), CD25 (clone M-A251), CD32 (clone FLI8.26), CD54 (clone HA58), 
1 CD62L (clone DREG-56), CD86 (clone 2331), CD184 (clone 12G5), HLA-A,B,C (clone G46-

2 2.6); Alexa Fluor 647- conjugated CD197 (clone 3D12) and isotype control antibodies: FITC-

3 conjugated mouse IgG1 (clone MOPC-21), IgG2a (clone G155- 178), IgG2b (clone MPC-11);

4 PE-conjugated mouse IgG1 (clone MOPC-21), IgG2a (clone G155-178), IgM (clone G155-228);

5 PerCPconjugated mouse IgG2a (clone X39); PE-Cy7-conjugated mouse IgG1 (clone MOPC-

6 21); APC-conjugated mouse IgG1 (clone MOPC- 21), IgG2a (clone G155-178), IgG2b (clone

7 27-35); Alexa Fluor 647 rat IgG2a (clone R35-95). PE-conjugated mouse monoclonal antibodies

8 against human CD282 (clone TL2.1) and CD284 (clone HTA125) and isotype control antibody

9 PE-conjugated mouse IgG2a (clone eBM2a) were obtained from eBioscience (San Diego, CA, 10 USA). Anti-human monoclonal blocking antibodies against TLR-1 (clone H2G2), TLR-2 (clone 11 B4H2), TLR-4 (clone W7C11), TLR-5 (clone Q2G4), TLR-6 (clone C5C8) and isotype control 12 human IgA2 (clone T9C6) and mouse IgG1 (clone T8E5) were purchased from Invivogen 13 (Toulouse, France).

14 HK mycobacterial preparations

\section{Blood collection}

Human peripheral blood was obtained by venipuncture from 36 Caucasian healthy volunteers and was collected into K2 ethylene diamine tetraacetic acid (K2-EDTA) vacutainer tubes (BD Biosciences). The study population had a mean age of $28 \pm 8.5$ years and included 14 females and 22 males. A written informed consent was obtained from each donor and the study protocol was approved by the Faculty Research Ethics Committees at Kingston University and University of Balamand.

\section{Stimulation of whole blood cultures}

Preliminary experiments using 3 and $6 \mathrm{~h}$ time points to stimulate whole blood with different concentrations $(30,100$ or $300 \mu \mathrm{g} / \mathrm{ml})$ of $\mathrm{HK} M$. vaccae or $M$. obuense preparations have revealed that the optimal regulation of surface receptors expression by either

31 mycobacterial preparation was observable at $300 \mu \mathrm{g} / \mathrm{ml}$ (Supplementary Figure 1A) and after 3

$32 \mathrm{~h}$ of stimulation (Supplementary Figure 1B). Therefore, these optimized stimulation conditions 
were employed in all investigations on the expression of surface receptors in which whole blood

2 cultures (total culture volume $=3 \mathrm{ml}$ ) were either left unstimulated or were stimulated at $37^{\circ} \mathrm{C}$

3 in a $5 \% \mathrm{CO} 2$ humidified atmosphere. A similar protocol was adopted for the analysis of

4 cytokines and chemokines levels, except that the stimulation period was extended to $24 \mathrm{~h}$.

5 Whole blood incubated with equivalent amounts of BBS (vehicle) served as unstimulated 6 control.

\section{Immunophenotyping of leukocytes}

After $3 \mathrm{~h}$ stimulation, blood leukocyte populations were immunophenotyped for a set of cell surface receptors. A volume of $100 \mu \mathrm{l}$ of whole blood was incubated for $30 \mathrm{~min}$ at room temperature (RT) in the dark with optimized concentrations of the previously mentioned antibodies or isotype control antibodies. Erythrocytes were then lysed by incubation with FACS lysing solution (BD Biosciences) for 10-15 min at RT in the dark. Leukocytes were collected by centrifugation at $350 \times \mathrm{g}$, for $5 \mathrm{~min}$ at RT and washed once with Cell Wash solution (BD Biosciences), then re-collected by centrifugation at $350 \times \mathrm{g}$, for $5 \mathrm{~min}$ at RT, and finally resuspended in Cell Fix solution (BD Biosciences). Fixed samples were run on a FACSCalibur flow cytometer (BD Biosciences) equipped with an argon ion laser $(488 \mathrm{~nm})$ and a red diode laser $(635 \mathrm{~nm})$ and the obtained data was analyzed by means of CellQuestPro software (BD Biosciences). A minimum of 20,000 cells were acquired per sample. The signals were obtained in a linear mode for forward scatter (FSC) and side scatter (SSC) channels and in a logarithmic mode for fluorescence channels. The intensity and color compensation were set by using Calibrite beads (BD Biosciences) in combination with FACSComp software (BD Biosciences), and by running single-color stained blood cells. Lymphocytes and neutrophils were identified by their FSC and SSC properties. Monocytes were identified by their $\mathrm{CD} 14^{+} / \mathrm{SSC}$ profile, whereas in TLR blocking experiments, monocytes were gated according to their FSC and SSC profile. Bivariate dot plots were generated to determine the percentage of cells positive for the particular surface receptor within a gated cell population. Histogram plots were used to indicate the density of cell surface receptors which were expressed as geometric mean fluorescence intensity (MFI) on receptor-positive cell population.

\section{Lymphocyte proliferation assay}

Peripheral blood was diluted with an equal volume of RPMI 1640 medium (Lonza, Slough, UK) supplemented with standard concentrations of L-glutamine (Lonza), penicillin and streptomycin (Lonza). Blood was then layered over Ficoll-Paque plus (GE Healthcare, Little 
1 Chalfont, UK) and centrifuged at $400 \times g$ for $35 \mathrm{~min}$ at $19{ }^{\circ} \mathrm{C}$. Peripheral blood mononuclear

2 cells (PBMCs) were then collected from the interface and were washed twice. Finally, PBMCs

3 were resuspended in RPMI medium supplemented with 10\% heat-inactivated pooled human AB

4 serum (Lonza). PBMCs were checked for viability ( $>95 \%$ ), using the trypan blue exclusion

5 method, were seeded, in triplicates, in 96-well flat-bottom plates (Corning, Tewksbury, USA) at

6 a density of $2 \times 10^{5}$ cells/well, and were stimulated in the presence or absence of increasing

7 concentrations of HK M. vaccae or M. obuense $(1,3,10,30,100$ and $300 \mu \mathrm{g} / \mathrm{ml})$ or $10 \mu \mathrm{g} / \mathrm{ml}$ of

8 Candida albicans (C. albicans) soluble antigen (Greer Laboratories, Lenoir, NC, USA). Cultures

9 were maintained for a period of 7 days at $37^{\circ} \mathrm{C}$ in a $5 \% \mathrm{CO} 2$ humidified incubator. On day 6 ,

10 PBMC cultures were pulsed with $1 \mu \mathrm{Ci} /$ well tritiated thymidine (Perkin Elmer, San Jose, CA,

11 USA) for $16 \mathrm{~h}$. Cells were then harvested onto glass fiber filter disks (Connectorate AG,

12 Dietikon, Switzerland) using a cell harvester (Inotech Biotechnologies, Basel, Switzerland) and

13 radioactivity was measured in a liquid scintillation counter (Perkin Elmer). Unstimulated cultures

14 lacking the antigen and containing equivalent amounts of BBS (vehicle) served as negative

15 controls. The radioactivity of incorporated thymidine was obtained as counts per minute (cpm).

16 Results are presented as stimulation index ( $\mathrm{SI})$, which is defined as follows: mean cpm of

17 antigen-stimulated cultures divided by the mean cpm of unstimulated cultures. A donor is considered as a responder to a given antigen if the $\mathrm{SI}$ is $\geq 3$.

\section{Determination of cytokines, chemokines and SCD62L levels}

The release of soluble mediators, chemokine C-X-C motif ligand 8 (CXCL8)/ IL-8, IFN- $\alpha$, IFN-y, IL-2, IL-4, IL-5, IL-6, IL-10, IL-12 (p70), IL-13, IL-23 (p19/p40), macrophage inflammatory protein 1 (MIP1)- $\alpha$, regulated on activation normal T cell expressed and secreted (RANTES), soluble (s) CD62L and TNF- $\alpha$, was measured in supernatants from whole blood cultures using ELISA (R\&D Systems, Abingdon, UK) in accordance with the manufacturer's instructions. Samples were tested in duplicates and the optical densities were read using Multiskan Ascent micro plate reader (Thermo, Waltham, MA, USA). In a restricted set of experiments, SCD62L levels were also assayed in supernatants of purified PBMCs that were cultured in RPMI medium supplemented with 10\% heat-inactivated fetal bovine serum (FBS; Lonza) and were left either unstimulated or stimulated with $300 \mu \mathrm{g} / \mathrm{ml}$ of HK M. vaccae or M. obuense for 3 and $6 \mathrm{~h}$.

\section{TLR blocking}

Whole blood was incubated with $10 \mu \mathrm{g} / \mathrm{ml}$ of cell culture grade, azide-free blocking 32 monoclonal antibodies against TLR1, TLR2, TLR4, TLR5, TLR6 or isotype control antibodies 
(listed above) for $1 \mathrm{~h}$ prior to stimulating whole blood with HK M. obuesne for $3 \mathrm{~h}$. As control

2 experiments for testing the efficiency of TLR blocking, specific TLR ligands (Invivogen) were

3 used. TLR agonists consisted of: Pam3CSk4 (300 ng/ml), HK Listeria monocytogenes $\left(10^{8}\right.$

4 cells $/ \mathrm{ml}$ ), lipopolysaccharide (LPS; purified from Escherichia coli $\mathrm{K} 12 ; 100 \mathrm{ng} / \mathrm{ml}$ ), flagellin

5 (purified from Bacillus subtilis; $10 \mu \mathrm{g} / \mathrm{ml}$ ) and FSL-1 (100 $\mathrm{ng} / \mathrm{ml}$ ) which signal through TLR2/1,

6 TLR2, TLR4, TLR5 and TLR2/6 respectively.

\section{Statistical analysis}

All statistical analyses were conducted by means of GraphPad Prism software (version 6; GraphPad Software, San Diego, CA, USA) by applying paired t-test, Wilcoxon signed rank test or Friedman test followed by Dunn's multiple comparison post hoc test. Data are expressed as mean values \pm standard error of the mean (SEM) and differences were considered to be statistically significant at $p$ values $<0.05$.

\section{Results}

M. vaccae and $M$. obuense modulate, in vitro, the expression of surface receptors on monocytes and neutrophils in whole blood

The expression levels of a panel of surface receptors on monocytes (Fig. 1, Table 1, Supplementary Table 1), neutrophils (Table 2, Supplementary Table 2) and lymphocytes in whole blood obtained from 15 donors and stimulated in vitro with $\mathrm{HK} M$. vaccae or $M$. obuense were evaluated. Monocytes and neutrophils exhibited substantial variations in the expression level of different categories of cell surface receptors following whole blood stimulation with $M$. vaccae or $M$. obuense. Among the adhesion receptors analyzed, a significant 6.5- and 5-fold remarkable reduction $(p<0.05)$ in the percentage of monocytes expressing CD62L was detected following stimulation with $M$. vaccae and $M$. obuense, respectively. This reduction in CD62L expression was also accompanied by a significant 4 -fold decrease $(p<0.05)$ in the MFI of CD62L (Fig. 1A, Table 1). Conversely, a 2.75-fold elevation $(p<0.05)$ in the percentage of monocytes expressing CD2 was observed following mycobacterial stimulation. Moreover, the intensity of receptor expression, measured as MFI, of CD18 (Fig. 1B), CD11a, CD44, CD54 and CD58 was significantly upregulated $(p<0.05)$ on monocytes post stimulation with either mycobacterial preparation (Table 1). On the other hand, both mycobacteria affected the expression of a restricted number of adhesion receptors on neutrophils. While the expression of

31 CD62L was significantly reduced, a significant increase $(p<0.05)$ in the expression of only

32 CD18 and CD54 could be detected (Table 2). 
Significant variations in the expression of antigen presentation and co-stimulatory receptors on monocytes and neutrophils subsequent to stimulation with mycobacterial 3 preparations were also detected. In unstimulated blood cultures, monocytes lacked the 4 expression of CD80, but stimulation with either mycobacterial preparation induced monocytes to 5 express CD80 (Table 1). A significant upregulation $(p<0.05)$ in the expression of CD86 (Fig.1A), HLA-DPDQDR (Fig. 1B) and CD45 was observed on monocytes (Table 1). We have also noted, using a smaller sample size $(\mathrm{n}=9)$, a significant increase $(p<0.05)$ in the percentage of $\mathrm{CD} 137 \mathrm{~L}^{+}$monocytes following stimulation with $M$. vaccae or $M$. obuense from $10 \pm 1.5 \%$ to $38 \pm 5 \%$ and $51 \pm 4.1 \%$, respectively. Stimulation with either mycobacterial preparation caused an increase in the MFI of CD45 on neutrophils; however, this increase was only statistically significant $(p<0.05)$ with $M$. vaccae. On the contrary, stimulation with $M$. vaccae, but not with $M$. obuense, significantly decreased $(p<0.05)$ the MFI of HLA-ABC on neutrophils (Table 2). Members of the pattern recognition receptors (PRRs), CD14, CD36, and CD206, displayed an upregulated expression $(p<0.05)$ on monocytes after stimulation with either mycobacterial preparation (Table 1). In a set of preliminary experiments conducted on 5 donors, both $M$. vaccae and $M$. obuense were able to highly up-regulate TLR expression on monocytes with approximately a 2-fold increase in the MFI of CD282 (TLR-2) (unstimulated: $77 \pm 8.4$; M. vaccae: $155 \pm 9.0$; M. obuense: $173 \pm 10 ; p<0.05$ vs. unstimulated) and approximately a 7-fold increase in the percentage of $\mathrm{CD}_{284^{+}}\left(\mathrm{TLR}-4^{+}\right)$monocytes (unstimulated: $8 \pm 1 \%$; $M$. vaccae: $53 \pm 6 \%$; $M$. obuense: $59 \pm 6 \% ; p<0.05$ vs. unstimulated). The expression of complement and Fc receptors was significantly modified on monocytes and neutrophils in response to stimulation with either mycobacterial preparation. A substantial increase $(p<0.05)$ in the expression of CD11C (Fig. 1B), CD11b and CD35 on monocytes was shown after stimulation with $M$. vaccae or $M$. obuense (Table 1). On the other hand, both mycobacterial preparations induced a modest but significant enhancement $(p<0.05)$ in the expression of CD64 and CD89 on monocytes (Table 1). On neutrophils, a significant elevation $(p<0.05)$ in the expression of CD11c, CD16, CD35 and CD64 was observed following stimulation with either mycobacterial preparation, whereas a significant elevation $(p<0.05)$ in the expression of CD11b and CD89 was only noted following stimulation with M. vaccae (Table 2).

Significant variations in the expression of cytokine and chemokine receptors, on monocytes and neutrophils, were detected following stimulation with either of the $\mathrm{HK}$ mycobacteria. Both preparations were equipotent at significantly upregulating $(p<0.05)$, on 
downregulating $(p<0.05)$ the expression of CD184 (Fig. 1A) and CD119 (Table 1) In

2 neutrophils, stimulation with either $M$. vaccae or $M$. obuense significantly reduced $(p<0.05)$ the 3 percentage of cells expressing CD184 and significantly downregulated $(p<0.05)$ the MFI of

4 CD119 (Table 2). In addition, when tested on a smaller sample of 9 donors, the MFI of CD114 5 was found to be downregulated following mycobacterial stimulation (unstimulated: $97 \pm 4 ; M$. 6 vaccae: $41 \pm 1 ; M$. obuense: $55 \pm 4)$. However, this effect only attained statistical significance $(p<$ 7 0.05) with $M$. vaccae. In this short-term in vitro assay, neither $M$. vaccae nor $M$. obuense 8 induced statistically significant variations in the expression level of any of the screened surface 9 receptors on lymphocytes (data not shown).

\section{TLR-1 and TLR-2 contribute to the M. obuense-induced upregulation of CD11C and HLA- DR expression on monocytes}

TLR-2 has been previously shown to play a role in mediating the main transcriptional responses occurring after DC stimulation with HK M. vaccae (Le Bert, et al. 2011). In order to examine whether TLR-2 and other TLRs (TLR-1, 4, 5 and 6) are involved in mediating the $M$. obuense-induced upregulation of surface receptors on monocytes in whole blood, TLR blocking experiments $(n=6)$ with specific antibodies against TLR-1, TLR-2, TLR-4, TLR-5 and TLR-6 were performed on the expression of one adhesion receptor (CD11c) and another receptor involved in antigen presentation (HLA-DR), both of which displayed significant variations on monocytes upon mycobacterial stimulation. The blocking capacity of these antibodies was initially confirmed in control experiments using stimulation with specific TLR ligands, as described in materials and methods (Fig. 2A).

Blocking either TLR-1 or TLR-2 significantly abrogated $(p<0.05)$ the $M$. obuense-induced upregulation of the two tested receptors (Fig. 2B). Analysis of combined blocking with anti-TLR1 and anti-TLR2, versus the blocking induced by either antibody separately, resulted in an improved blocking effect which reached statistical significance $(p<0.05)$ versus either antibody in the case of CD11c, but only against anti-TLR2 in the case of HLA-DR. However, simultaneous blocking of TLR-2 and TLR- 6 was comparable to the effect observed with blocking TLR-2 alone in reversing the $M$. obuense-induced expression of CD11C and HLA-DR (Fig. 2B). Blocking of TLR-4, 5 and 6 did not cause any significant reduction in the $M$. obuense-induced upregulation of CD11C and HLA-DR (Fig. 2B). Taken together, these results indicated a major contribution for both TLR-1 and TLR-2 in mediating the M. obuense-induced regulation of CD11c and HLA-DR expression on monocytes. 

specific set of cytokines and chemokines

A panel of 12 cytokines and 3 chemokines was analyzed in supernatants of whole blood cultures from 15 different donors that were stimulated with $M$. vaccae or $M$. obuense for a period of $24 \mathrm{~h}$. Among the 12 screened cytokines, IL-6 and TNF- $\alpha$ levels were markedly increased ( $p<$ 0.05 ) in response to stimulation with $M$. vaccae or $M$. obuense, whereas IL-10 levels were significantly elevated $(p<0.05)$ only after stimulation with $M$. vaccae (Fig. 3 ). The secreted levels of the 3 studied chemokines also showed a significant increase following mycobacterial stimulation. A 45- and 42-fold increase $(p<0.05)$ in CXCL8/IL-8 levels was detected after stimulation with $M$. vaccae and $M$. obuense, respectively (Fig. 3). Moreover, blood stimulation with $M$. vaccae or $M$. obuense significantly induced $(p<0.05)$ MIP-1a production (Fig. 3). Likewise, RANTES levels were increased in response to stimulation with $M$. vaccae or $M$. obuense; however, this increase attained statistical significance $(p<0.05)$ only for the latter (Fig. 3). No significant difference in IL-18 levels was observed between unstimulated $(612 \pm 71$ $\mathrm{pg} / \mathrm{ml})$ and $M$. vaccae- $(638 \pm 65 \mathrm{pg} / \mathrm{ml})$ or $M$. obuense- $(641 \pm 70 \mathrm{pg} / \mathrm{ml})$ stimulated blood cultures. Moreover, IFN- $\alpha$, IFN- $y$, IL-2, IL-4, IL-5, IL-12 (p70), IL-13 and IL-23 (p19/p40) levels in unstimulated and mycobacteria-stimulated blood cultures were below the detection limit of the employed assays.

In order to determine whether the observed $M$. vaccae- and $M$. obuense-induced decrease in CD62L surface expression was associated with an increase in the release of SCD62L, supernatants from unstimulated and mycobacteria-stimulated whole blood cultures were collected. There was no significant difference $(p>0.05)$ in SCD62L levels in culture supernatants after 3, 6 or $24 \mathrm{~h}$ of mycobacterial stimulation when compared with their respective unstimulated controls (Supplementary Figure 2). Moreover, using purified PBMC cultures from 6 different donors, we could not detect SCD62L levels in the supernatants of unstimulated or mycobacteria-stimulated PBMCs tested after 3 and $6 \mathrm{~h}$ culture periods.

\section{M. vaccae and $M$. obuense induce proliferative responses of PBMCs}

In order to determine the optimal concentrations of HK M. vaccae and M. obuense that are capable of inducing strong proliferative responses in PBMCs from healthy donors, different concentrations $(1,3,10,30,100$ and $300 \mu \mathrm{g} / \mathrm{ml})$ of either mycobacterial preparation were tested on 4 separate donors (Fig. 4A). At the lowest and highest concentrations, only weak or no

32 responses could be detected to either preparation (Fig. 4A). To further verify our data, PBMCs 33 from 8 additional donors were tested at concentrations ranging from $3-100 \mu \mathrm{g} / \mathrm{ml}$. Results of 
1 proliferative responses from the 12 different donors tested revealed that the highest proliferative

2 responses to $M$. obuense were induced at $3 \mu \mathrm{g} / \mathrm{ml}$ (Fig. 4B), which attained a statistically 3 significant difference $(p<0.05)$ versus 30 or $100 \mu \mathrm{g} / \mathrm{ml}$. In contrast, maximal responses to $M$.

4 vaccae, noted at $10 \mu \mathrm{g} / \mathrm{ml}$, were nearly equivalent to those induced by $30 \mu \mathrm{g} / \mathrm{ml}$ and were 5 significantly higher $(p<0.05)$ than those induced by $100 \mu \mathrm{g} / \mathrm{ml}$. At the $3 \mu \mathrm{g} / \mathrm{ml}$ concentration, the

$6 M$. obuense-induced proliferative responses were significantly higher $(p<0.05)$ than the 7 responses induced by $M$. vaccae, whereas the reverse was true at the $30 \mu \mathrm{g} / \mathrm{ml}$ concentration 8 (Fig. 4B). Soluble recall antigen of $C$. albicans $(10 \mu \mathrm{g} / \mathrm{ml})$ was included in the proliferation 9 assays as a positive control on account of its known potency to induce strong PBMC 10 proliferative responses. PBMCs from all tested donors showed high proliferative responses to $C$. 11 albicans (Mean $\mathrm{SI}=134 \pm 39)$ and these responses were significantly higher $(p<0.05)$ than 12 those induced by $M$. vaccae or M. obuense.

\section{Discussion} step in the process of assessing their immunomodulatory properties for potential immunotherapeutic applications. Our study is the first to utilize the established human whole blood model (Darcissac, et al. 1996a) to investigate the capacity of HK $M$. vaccae and $M$. obuense preparations to modulate the expression of an array of different categories of cell surface receptors expressed on human monocytes, neutrophils and lymphocytes. The reason behind employing the whole blood model in our study is that it closely resembles the status of circulating cells in vivo and it involves physiological concentrations of factors that affect leukocytes function. Our immunophenotyping results demonstrated that under the experimental conditions used, the target peripheral blood immune cells for HK $M$. vaccae and $M$. obuense appear to be the phagocytic cells; namely, neutrophils and monocytes. Whole blood stimulation with either mycobacterial preparation upregulated the expression of the adhesion receptors, CD2, CD11a, CD44 and CD58 on monocytes and of CD18 and CD54 on monocytes and neutrophils. These findings are similar to those previously reported following whole blood stimulation with the mycobacterial cell wall component muramyl dipeptide (MDP) or with endotoxin (Darcissac, et al. 1996a). The increased expression of CD2, and more importantly of its ligand CD58 on monocytes, may indicate an increased ability of monocyte-macrophage cells to adhere to T lymphocytes which constitutively express the CD2 receptor (Lopez, et al. 2001). Accordingly, the immunomodulating activity of mycobacterial preparations may depend on their ability to regulate CD58 expression on antigen presenting cells (APCs), thereby enhancing 
antigen presentation and induction of adaptive immunity (Wingren, et al. 1995). Similarly, increased expression of CD54 on APCs may contribute to antigen-specific T cell activation by enhancing the adhesion of T cells to APCs (Gaglia, et al. 2000). The most intriguing observation was the dramatic reduction in the expression of L-selectin (CD62L) on monocytes, and to a lesser extent on neutrophils, following mycobacterial stimulation. This observation is in line with previous reports which demonstrated that in response to activation signals, L-selectin is shed from the surface of neutrophils and monocytes within minutes (Janke, et al. 2009; Nijhuis, et al. 2007). Upon the ectodomain shedding of $C D 62 L$, its soluble form, $S C D 62 L$, is released into the extracellular environment (Ivetic 2013; Smalley and Ley 2005). Following whole blood stimulation with either mycobacterial preparation, we could not detect any significant increase in SCD62L levels. Nevertheless, the constitutive levels of SCD62L detected in all supernatants were quite high and exceeded $1000 \mathrm{ng} / \mathrm{ml}$. Thus it is quite likely that such high constitutive levels of SCD62L could mask the detection of low levels of SCD62L shedding induced by mycobacterial stimulation (Schleiffenbaum, et al. 1992). In addition, our inability to detect SCD62L in unstimulated and mycobacteria-stimulated PBMC culture supernatants, despite the significant downregulation of $\mathrm{CD} 62 \mathrm{~L}$ expression on monocytes, might be attributed to low amounts of shed CD62L which are below the detection limit of our assay $(0.3 \mathrm{ng} / \mathrm{mL})$.

On the other hand, whole blood stimulation with $M$. vaccae or M. obuense has resulted in increased monocyte expression of key receptors involved in antigen presentation and costimulation including MHC-class II, CD80, CD86 and CD137L receptors. The observed upregulated expression of antigen presentation and costimulatory receptors strongly suggests a better antigen presentation function of mycobacteria-stimulated APC leading to improved T cell activation and survival (Gramaglia, et al. 2000). In fact, one of the mechanisms by which tumor cells escape immune detection is by down-regulating antigen presentation and costimulatory receptors which would generate anergic $T$ cells incapable of initiating an effective antitumor immune response (Driessens, et al. 2009; Thibodeau, et al. 2012). Consequently, augmenting the expression of costimulatory receptors might contribute toward enhanced antitumor immunity (Capece, et al. 2012).

We have also shown that mycobacterial stimulation was associated with a significant upregulation of different PRRs, including TLR-2, TLR-4, CD14, CD36 and CD206, on monocytes. This is the first description of an induced expression of CD206 on monocytes in a whole blood model and after a short period of mycobacterial stimulation. Previous studies have demonstrated extremely low or no CD206 expression on resting monocytes, with the CD206 
expression being restricted to immature DCs and macrophages (Kato, et al. 2000; Noorman, et

2 al. 1997; Wollenberg, et al. 2002). Therefore, it is likely that mycobacterial stimulation could 3 initiate the expression of markers on monocytes associated with a subsequent potential 4 differentiation of these cells into macrophages. The elevated CD14 and CD282 (TLR-2) gene 5 expression in mycobacteria-stimulated monocytes has been previously reported in $M$. vaccae6 stimulated THP-1 human monocytic cell line (Martinelli, et al. 2004). Such an effect could well 7 contribute towards boosting the recognition of pathogen-associated molecular patterns. On the 8 contrary, some studies have reported a significant downregulation of CD14 expression on 9 purified monocytes that were stimulated with live $M$. tuberculosis or HK $M$. avium- $M$. 10 intracellulare complex for 16 or $48 \mathrm{~h}$, respectively (Santucci, et al. 2000; Tsuyuguchi, et al. 11 1990). The apparent contradiction in CD14 expression between our study and other studies 12 previously reported might be attributed to differences in the culture systems, in the nature of 13 stimulus, and/or in the stimulation periods utilized.

Moreover, both mycobacterial preparations demonstrated an evident potential to upregulate the expression of complement and Fc-receptors on monocytes and neutrophils, an effect that is tightly linked to improved phagocytosis and immune complex clearance (Nimmerjahn and Ravetch 2008; Zipfel and Skerka 2009). For instance, in monoclonal antibodymediated therapy targeting tumor-associated antigens, upregulating the expression of complement and Fc receptors might enhance the process of tumor cell killing through antibodydependent cell-mediated cytotoxicity or complement-dependent cytotoxicity, respectively (Hogarth and Pietersz 2012; van Egmond and Bakema 2013).

Although both mycobacterial preparations altered the expression of various surface receptors on neutrophils and monocytes, no significant effect could be observed on lymphocytes. The lack of a modulatory effect by both mycobacterial preparations on lymphocytes might be attributed to the short $3 \mathrm{~h}$ stimulation period adopted in our whole blood culture system. Using purified PBMCs and longer stimulation periods of 24 and $48 \mathrm{~h}$, a previous study has shown that, in vitro, both HK M. vaccae and $M$. obuense upregulated the surface expression of various activation receptors on the $\gamma \delta \mathrm{T}$ lymphocyte subpopulation (Fowler, et al. 2012). Moreover, in vivo treatment with these HK mycobacterial preparations has been reported to induce, few weeks later, a measurable activation of cytotoxic $\mathrm{T}$ lymphocytes (Elia, et al. 2013). Another potential explanation is that blood lymphocytes fail to recognize both mycobacterial preparations due to the absence, or barely detectable surface expression of TLR2 which is a crucial receptor for the recognition of mycobacteria (Flo, et al. 2001). This 
explanation is further substantiated by the inability to detect the surface expression of TLR-2 on

2 lymphocytes in our system (data not shown).

Mycobacteria are known to trigger host immune responses following initial interaction with innate immune cells through selected PRRs (Killick, et al. 2013; Kleinnijenhuis, et al. 2011). A HK preparation of $M$. vaccae has been previously reported to induce in vitro DC activation via TLR2 (Le Bert, et al. 2011). To determine whether the interaction between HK M. obuense and TLRs was responsible for the increased surface expression of receptors on monocytes, blocking experiments with antibodies against TLR-1, 2, 4, 5 and 6 were carried out. These experiments clearly indicated that in human whole blood, blocking of TLR-1 and/or TLR-2 significantly inhibited the $M$. obuense-induced upregulation of CD11C and HLA-DR surface expression on monocytes. Despite the blocking of TLR-1 and/or TLR-2, the expression levels of CD11c and HLA-DR remained higher than those of unstimulated monocytes $(p<0.05)$ and therefore a possible participation of other receptors, such as nucleotide oligomerisation domain 2 (Nod-2) receptor, in mediating the biological effects of $M$. obuense cannot be excluded (Behr and Divangahi 2015). Differences in the average MFI values of MHC class II between TLR-blocking experiments (Fig. 2B) and immunophenotyping experiments (Table 1) can be explained by the fact that in TLR-blocking experiments, we used an antibody specific for only HLA-DR whereas in the immunophenotyping experiments an antibody recognizing the three MHC class II antigens, namely HLA-DPDQDR, was employed.

In the current work, we have measured a battery of cytokines and chemokines in supernatants of whole-blood cultures following $24 \mathrm{~h}$ of whole blood stimulation with $M$. vaccae or $M$. obuense. Among the cytokines tested, IL-6, IL-10, and TNF- $\alpha$ were significantly elevated following mycobacterial stimulation. This is in line with previous reports indicating the release of considerable amounts of IL-6, IL-10, and TNF- $\alpha$ upon activation of monocytes (Damsgaard, et al. 2009), the ability of $M$. vaccae to induce IL-10 and TNF- $\alpha$ production in cultures of isolated monocytes (Baran, et al. 2004), and increased secretion of IL-10 and TNF- $\alpha$ in PBMC cultures stimulated with HK M. bovis (Moreira, et al. 2012). Moreover, mycobacterial stimulation enhanced the production of the three tested chemokines: CXCL8/IL-8 and MIP-1 $\alpha$. In contrast, whole blood stimulation with $\mathrm{HK}$ mycobacteria did not induce the release of any of the signature T cell cytokines, IFN- $y$, IL-2 or IL-4. Thus, the profile of HK mycobacteria-induced cytokines and chemokines in the in vitro system used is most likely monocyte-derived and triggered by structures common to the two organisms. Among such structures are cell wall components containing muramyl peptides as well as heat shock proteins (HSPs) which have been reported 
1 to induce a range of proinflammatory cytokines and chemokines either in vitro or in vivo (Asea,

2 et al. 2000; Bahr, et al. 2003; Darcissac, et al. 1996b; Stanford, et al. 2009; Wang, et al. 2002).

3 This profile of cytokine release, dominated by IL-10 and proinflammatory cytokines, was also

4 reported following whole blood stimulation with HK Lactobacillus rhamnosus, Helicobacter

5 pylori, Burkholderia pseudomallei, MDP, and LPS (Darcissac, et al. 1996b; Duffy, et al. 2014;

6 Myers, et al. 2014). On the other hand, the release of cytokines that play a direct role in the

7 adaptive immune system has been reported to occur in whole blood cultures from healthy

8 donors after stimulation with certain gram-negative and gram-positive HK bacteria, with fungi,

9 with live BCG or with live M. intracelluare, but not with HK M. tuberculosis (Duffy, et al. 2014;

10 Kartalija, et al. 2013; Popa, et al. 2009; Reichenbach, et al. 2006). It is noteworthy to mention

11 that the cytokine responses to mycobacterial stimulation observed in the whole blood assay

12 might differ from those detected in other culture systems. While the levels of some cytokines

13 such as IL-2, IL-5, IL-6, IL-7, IL-13, TNF- $\alpha$, MCP-1, MIP-1 $\beta$ (Silva, et al. 2013) and IFN- $\gamma$ (Antas,

14 et al. 2004); Deenadayalan, et al. 2013; Silva, et al. 2013) were comparable between whole

15 blood and PBMC cultures stimulated with specific $M$. tuberculosis antigens, levels of other 16 cytokines like IL-1ß, IL-4, IL-10, IL-12 (p70), IL-17, G-CSF and GM-CSF were significantly 17 different between the two culture models (Silva, et al. 2013).

We have also examined the lymphoproliferative responses to HK $M$. vaccae and $M$. obuense through stimulation of PBMCs from healthy individuals. Distinct from cell proliferation occurring in cancers and other pathological processes, this in vitro assay is routinely used to assess the presence of mycobacteria-sensitized lymphocytes in peripheral blood and their ability to proliferate in response to mycobacterial antigens. Taking into consideration that $M$. vaccae and $M$. obuense are phylogenetically related, lack group iii antigens, possess their own species-specific group iv antigens, and are rich in group i common mycobacterial antigens (Stanford, et al. 2009), it can be assumed that the observed M. vaccae and M. obuense induced PBMC proliferative responses might be directed, mainly, against the common mycobacterial antigens. Previous studies, conducted to analyze the sensitization to environmental mycobacteria in north Lebanon, southeast England, and northern Malawi by skin testing and/or whole blood IFN- $\gamma$ release assay, revealed a very low level of exposure to some environmental mycobacteria including M. vaccae (Bahr, et al. 1986; Black, et al. 2001; Weir, et al. 2003).

Moreover, the healthy donors tested were not previously vaccinated with BCG in line with the policy adopted by the local health authorities. However, several studies have indicated that different mycobacterial species possess cross-reacting T-cell epitopes which could induce 
1 the proliferation of sensitized T-cell clones (Oftung, et al. 1998; Sieling, et al. 2005). Therefore,

2 M. vaccae- and M. obuense- specific T cells are likely to exhibit a broad spectrum of cross-

3 reactivity with an as yet to be identified environmental mycobacterial species that could be

4 abundant in the Lebanese environment and could elicit the observed lymphoproliferative

5 responses.

\section{7}

\section{Conflict of interest} 32 The authors declare they have no conflicts of interest. such as the adjuvant, the anti-infectious, and the anti-tumor effects

\section{Authors' contribution} analysis tools. SB and GMB wrote the paper.

As a limitation of the current study, our preliminary kinetics experiments were based on determining the optimal regulation of two surface receptors, and only at two time points, in response to mycobacterial stimulation. However, other receptors analyzed in this study might display optimal regulation at different stimulation periods. An additional limitation of our study was the analysis of cytokine levels at a single time point following mycobacterial stimulation of whole blood. Previous studies have emphasized the importance of identifying optimal stimulation time points correlating with peak levels of cytokines since these time points are cytokine- and stimulus-dependent (Hermann, et al. 2003; Lagrelius, et al. 2006). For instance, we could not detect IFN-y levels in $24 \mathrm{~h}$ mycobacteria-stimulated whole blood cultures. However, other studies employing longer stimulation periods of 5 and 6 days were capable of detecting IFN- $y$ in whole blood cultures stimulated with specific M. tuberculosis antigens (Antas, et al. 2004; Deenadayalan, et al. 2013; Silva, et al. 2013). Thus, future kinetic studies employing additional and extended time points might be useful in addressing the effect of mycobacterial stimulation on the expression of other surface receptors and on the release of cytokines.

In conclusion, our study revealed a potent ability of HK cell preparations of $M$. vaccae and $M$. obuense to regulate the expression of several receptors on phagocytic cells, and to induce the release of a specific set of cytokines and chemokines in this whole blood in vitro system. The data provided could serve as a baseline to explain the outcome of interaction between HK mycobacterial preparations and immune cells which in additional future studies might be correlated with the previously known in vivo activities of $\mathrm{HK}$ mycobacterial preparations

$\mathrm{SB}$ and GMB conceived and designed the experiments. SB performed the experiments. SB, $\mathrm{HM}, \mathrm{SM}, \mathrm{CA}$ and GMB analyzed the data. HM, SM and CA contributed reagents, materials, and 
2 We are grateful to Emale El Darzi and Jeannette El Tom for their technical assistance. This

3 work was supported by a special grant from the University of Balamand to GMB.

4

\section{References}

Abbot, N.C., Beck, J.S., Feval, F., Weiss, F., Mobayen, M.H., Ghazi-Saidi, K., et al., 2002. Immunotherapy with Mycobacterium vaccae and peripheral blood flow in long-treated leprosy patients, a randomised, placebo-controlled trial. Eur. J. Vasc. Endovasc. Surg. 24 202-208.

Adams, V.C., Hunt, J.R., Martinelli, R., Palmer, R., Rook, G.A., Brunet, L.R., 2004. Mycobacterium vaccae induces a population of pulmonary CD11c+ cells with regulatory potential in allergic mice. Eur. J. Immunol. 34 631-638.

Antas, P.R., Cardoso, F.L., Oliveira, E.B., Gomes, P.K., Cunha, K.S., Sarno, E.N., et al., 2004. Whole blood assay to access T cell-immune responses to Mycobacterium tuberculosis antigens in healthy Brazilian individuals. Mem. Inst. Oswaldo Cruz 99 53-55.

Arkwright, P.D., David, T.J., 2001. Intradermal administration of a killed Mycobacterium vaccae suspension (SRL 172) is associated with improvement in atopic dermatitis in children with moderate-to-severe disease. J. Allergy Clin. Immunol. 107 531-534.

Asea, A., Kraeft, S.K., Kurt-Jones, E.A., Stevenson, M.A., Chen, L.B., Finberg, R.W., et al., 2000. HSP70 stimulates cytokine production through a CD14-dependant pathway, demonstrating its dual role as a chaperone and cytokine. Nat. Med. 6 435-442.

Bahr, G.M., De La Tribonniere, X., Darcissac, E., Ajana, F., Bocket, L., Sissoko, D., et al., 2003. Clinical and immunological effects of a 6 week immunotherapy cycle with murabutide in HIV-1 patients with unsuccessful long-term antiretroviral treatment. J. Antimicrob. Chemother. 51 1377-1388.

Bahr, G.M., Stanford, J.L., Rook, G.A., Rees, R.J., Frayha, G.J., Abdelnoor, A.M., 1986. Skin sensitization to mycobacteria amongst school children prior to a study of BCG vaccination in North Lebanon. Tubercle 67 197-203.

Baran, J., Baj-Krzyworzeka, M., Weglarczyk, K., Ruggiero, I., Zembala, M., 2004. Modulation of monocyte-tumour cell interactions by Mycobacterium vaccae. Cancer Immunol. Immunother. 53 1127-1134.

Barclay, A.N., Brown, M.H., Law, S.K.A., McKnight, A.I., Tomhnson, M.G., van der Merwe, P., 1997. The leucocyte antigen facts book, 2nd ed. Academic Press, San Diego.

Behr, M.A., Divangahi, M., 2015. Freund's adjuvant, NOD2 and mycobacteria. Curr. Opin. Microbiol. 23 126-132. 
Black, G.F., Dockrell, H.M., Crampin, A.C., Floyd, S., Weir, R.E., Bliss, L., et al., 2001. Patterns and implications of naturally acquired immune responses to environmental and tuberculous mycobacterial antigens in northern Malawi. J. Infect. Dis. 184 322-329.

Camporota, L., Corkhill, A., Long, H., Lordan, J., Stanciu, L., Tuckwell, N., et al., 2003. The effects of Mycobacterium vaccae on allergen-induced airway responses in atopic asthma. Eur. Respir. J. 21 287-293.

Cananzi, F.C., Mudan, S., Dunne, M., Belonwu, N., Dalgleish, A.G., 2013. Long-term survival and outcome of patients originally given Mycobacterium vaccae for metastatic malignant melanoma. Hum. Vaccin Immunother. 9 2427-2433.

Capece, D., Verzella, D., Fischietti, M., Zazzeroni, F., Alesse, E., 2012. Targeting costimulatory molecules to improve antitumor immunity. J. Biomed. Biotechnol. 2012926321.

Dalgleish, A.G. and The IMAGE I Trial Investigators, 2015. A multicenter randomized, openlabel, proof-of-concept, phase II trial comparing gemcitabine with and without IMM-101 in advanced pancreatic cancer, Anonymous 2015, pp. 336.

Damsgaard, C.T., Lauritzen, L., Calder, P.C., Kjaer, T.M., Frokiaer, H., 2009. Whole-blood culture is a valid low-cost method to measure monocytic cytokines - a comparison of cytokine production in cultures of human whole-blood, mononuclear cells and monocytes. J. Immunol. Methods 340 95-101.

Darcissac, E.C., Bahr, G.M., Parant, M.A., Chedid, L.A., Riveau, G.J., 1996a. Selective induction of CD11a,b,c/CD18 and CD54 expression at the cell surface of human leukocytes by muramyl peptides. Cell. Immunol. 169 294-301.

Darcissac, E.C., Bahr, G.M., Pouillart, P.R., Riveau, G.J., Parant, M.A., 1996b. Selective potentiation of cytokine expression in human whole blood by murabutide, a muramyl dipeptide analogue. Cytokine 8 658-666.

Deenadayalan, A., Maddineni, P., Raja, A., 2013. Comparison of whole blood and PBMC assays for T-cell functional analysis. BMC Res. Notes 6 120-0500-6-120.

Dlugovitzky, D., Fiorenza, G., Farroni, M., Bogue, C., Stanford, C., Stanford, J., 2006. Immunological consequences of three doses of heat-killed Mycobacterium vaccae in the immunotherapy of tuberculosis. Respir. Med. 100 1079-1087.

Driessens, G., Kline, J., Gajewski, T.F., 2009. Costimulatory and coinhibitory receptors in antitumor immunity. Immunol. Rev. 229 126-144.

Duffy, D., Rouilly, V., Libri, V., Hasan, M., Beitz, B., David, M., et al., 2014. Functional analysis via standardized whole-blood stimulation systems defines the boundaries of a healthy immune response to complex stimuli. Immunity 40 436-450.

Eaton, J.D., Perry, M.J., Nicholson, S., Guckian, M., Russell, N., Whelan, M., et al., 2002. Allogeneic whole-cell vaccine: a phase $\mathrm{I} / \mathrm{Il}$ study in men with hormone-refractory prostate cancer. BJU Int. 89 19-26. 
Elia, A., Lincoln, L., Brunet, L., Hagemann, T., 2013. Treatment with IMM-101 induces protective CD8+ T cell responses in clinically relevant models of pancreatic cancer. Journal for ImmunoTherapy of Cancer 1 P215.

Flo, T.H., Halaas, O., Torp, S., Ryan, L., Lien, E., Dybdahl, B., et al., 2001. Differential expression of Toll-like receptor 2 in human cells. J. Leukoc. Biol. 69 474-481.

Fowler, D.W., Copier, J., Wilson, N., Dalgleish, A.G., Bodman-Smith, M.D., 2012. Mycobacteria activate gammadelta T-cell anti-tumour responses via cytokines from type 1 myeloid dendritic cells: a mechanism of action for cancer immunotherapy. Cancer Immunol. Immunother. 61 535547.

Gaglia, J.L., Greenfield, E.A., Mattoo, A., Sharpe, A.H., Freeman, G.J., Kuchroo, V.K., 2000. Intercellular adhesion molecule 1 is critical for activation of CD28-deficient T cells. J. Immunol. 165 6091-6098.

Gramaglia, I., Cooper, D., Miner, K.T., Kwon, B.S., Croft, M., 2000. Co-stimulation of antigenspecific CD4 T cells by 4-1BB ligand. Eur. J. Immunol. 30 392-402.

Hermann, C., von Aulock, S., Graf, K., Hartung, T., 2003. A model of human whole blood lymphokine release for in vitro and ex vivo use. J. Immunol. Methods 275 69-79.

Hogarth, P.M., Pietersz, G.A., 2012. Fc receptor-targeted therapies for the treatment of inflammation, cancer and beyond. Nat. Rev. Drug Discov. 11 311-331.

Ivetic, A., 2013. Signals regulating L-selectin-dependent leucocyte adhesion and transmigration. Int. J. Biochem. Cell Biol. 45 550-555.

Janke, M., Poth, J., Wimmenauer, V., Giese, T., Coch, C., Barchet, W., et al., 2009. Selective and direct activation of human neutrophils but not eosinophils by Toll-like receptor 8 . J. Allergy Clin. Immunol. 123 1026-1033.

Johnson, J.L., Kamya, R.M., Okwera, A., Loughlin, A.M., Nyole, S., Hom, D.L., et al., 2000. Randomized controlled trial of Mycobacterium vaccae immunotherapy in non-human immunodeficiency virus-infected ugandan adults with newly diagnosed pulmonary tuberculosis. The Uganda-Case Western Reserve University Research Collaboration. J. Infect. Dis. 181 1304-1312.

Kartalija, M., Ovrutsky, A.R., Bryan, C.L., Pott, G.B., Fantuzzi, G., Thomas, J., et al., 2013. Patients with nontuberculous mycobacterial lung disease exhibit unique body and immune phenotypes. Am. J. Respir. Crit. Care Med. 187 197-205.

Kassa, D., Ran, L., Geberemeskel, W., Tebeje, M., Alemu, A., Selase, A., et al., 2012. Analysis of immune responses against a wide range of Mycobacterium tuberculosis antigens in patients with active pulmonary tuberculosis. Clin. Vaccine Immunol. 19 1907-1915.

Kato, M., Neil, T.K., Fearnley, D.B., McLellan, A.D., Vuckovic, S., Hart, D.N., 2000. Expression of multilectin receptors and comparative FITC-dextran uptake by human dendritic cells. Int. Immunol. 12 1511-1519. 
Kawai, K., Miyazaki, J., Joraku, A., Nishiyama, H., Akaza, H., 2013. Bacillus Calmette-Guerin (BCG) immunotherapy for bladder cancer: current understanding and perspectives on engineered BCG vaccine. Cancer. Sci. 104 22-27.

Killick, K.E., Ni Cheallaigh, C., O'Farrelly, C., Hokamp, K., MacHugh, D.E., Harris, J., 2013.

Receptor-mediated recognition of mycobacterial pathogens. Cell. Microbiol. 15 1484-1495.

Kleinnijenhuis, J., Oosting, M., Joosten, L.A., Netea, M.G., Van Crevel, R., 2011. Innate immune recognition of Mycobacterium tuberculosis. Clin. Dev. Immunol. 2011405310.

Lagrelius, M., Jones, P., Franck, K., Gaines, H., 2006. Cytokine detection by multiplex technology useful for assessing antigen specific cytokine profiles and kinetics in whole blood cultured up to seven days. Cytokine 33 156-165.

Le Bert, N., Chain, B.M., Rook, G., Noursadeghi, M., 2011. DC priming by M. vaccae inhibits Th2 responses in contrast to specific TLR2 priming and is associated with selective activation of the CREB pathway. PLoS One 6 e18346.

Lehrer, A., Bressanelli, A., Wachsmann, V., Bottasso, O., Bay, M.L., Singh, M., et al., 1998. Immunotherapy with Mycobacterium vaccae in the treatment of psoriasis. FEMS Immunol. Med. Microbiol. 21 71-77.

Lopez, R.D., Waller, E.K., Lu, P.H., Negrin, R.S., 2001. CD58/LFA-3 and IL-12 provided by activated monocytes are critical in the in vitro expansion of CD56+ T cells. Cancer Immunol. Immunother. 49 629-640.

Martinelli, R., Adams, V.C., Rook, G.A.W., Brunet, L.R., 2004. Effects of Mycobacterium vaccae on toll-like receptor expression. J. Allergy Clin. Immunol. 113 S187.

Moreira, J., Aragao-Filho, W.C., Barillas, S.G., Barbosa, S.M., Pedroza, L.A., Condino-Neto, A., 2012. Human leucocytes response to viable, extended freeze-drying or heat-killed Mycobacterium bovis bacillus Calmette-Guerin. Scand. J. Immunol. 75 96-101.

Myers, N.D., Chantratita, N., Berrington, W.R., Chierakul, W., Limmathurotsakul, D., Wuthiekanun, V., et al., 2014. The role of NOD2 in murine and human melioidosis. J. Immunol. 192 300-307.

Nijhuis, M.M., Pasterkamp, G., Sluis, N.I., de Kleijn, D.P., Laman, J.D., Ulfman, L.H., 2007. Peptidoglycan increases firm adhesion of monocytes under flow conditions and primes monocyte chemotaxis. J. Vasc. Res. 44 214-222.

Nimmerjahn, F., Ravetch, J.V., 2008. Fcgamma receptors as regulators of immune responses. Nat. Rev. Immunol. 8 34-47.

Noorman, F., Braat, E.A., Barrett-Bergshoeff, M., Barbe, E., van Leeuwen, A., Lindeman, J., et al., 1997. Monoclonal antibodies against the human mannose receptor as a specific marker in flow cytometry and immunohistochemistry for macrophages. J. Leukoc. Biol. 61 63-72. 
O'Brien, M.E., Anderson, H., Kaukel, E., O'Byrne, K., Pawlicki, M., Von Pawel, J., et al., 2004. SRL172 (killed Mycobacterium vaccae) in addition to standard chemotherapy improves quality of life without affecting survival, in patients with advanced non-small-cell lung cancer: phase III results. Ann. Oncol. 15 906-914.

Oftung, F., Borka, E., Kvalheim, G., Mustafa, A.S., 1998. Mycobacterial crossreactivity of M. tuberculosis reactive $T$ cell clones from naturally converted PPD positive healthy subjects. FEMS Immunol. Med. Microbiol. 20 231-238.

Patel, P.M., Sim, S., O'Donnell, D.O., Protheroe, A., Beirne, D., Stanley, A., et al., 2008. An evaluation of a preparation of Mycobacterium vaccae (SRL172) as an immunotherapeutic agent in renal cancer. Eur. J. Cancer 44 216-223.

Popa, C., Barrera, P., Joosten, L.A., van Riel, P.L., Kullberg, B.J., van der Meer, J.W., et al., 2009. Cytokine production from stimulated whole blood cultures in rheumatoid arthritis patients treated with various TNF blocking agents. Eur. Cytokine Netw. 20 88-93.

Reichenbach, J., Schubert, R., Feinberg, J., Beck, O., Rosewich, M., Rose, M.A., et al., 2006. Impaired interferon-gamma production in response to live bacteria and Toll-like receptor agonists in patients with ataxia telangiectasia. Clin. Exp. Immunol. 146 381-389.

Romano, M., Huygen, K., 2012. An update on vaccines for tuberculosis - there is more to it than just waning of BCG efficacy with time. Expert Opin. Biol. Ther. 12 1601-1610.

Rook, G.A., Adams, V., Hunt, J., Palmer, R., Martinelli, R., Brunet, L.R., 2004. Mycobacteria and other environmental organisms as immunomodulators for immunoregulatory disorders. Springer Semin. Immunopathol. 25 237-255.

Santucci, M.B., Amicosante, M., Cicconi, R., Montesano, C., Casarini, M., Giosue, S., et al., 2000. Mycobacterium tuberculosis-induced apoptosis in monocytes/macrophages: early membrane modifications and intracellular mycobacterial viability. J. Infect. Dis. 181 1506-1509.

Schleiffenbaum, B., Spertini, O., Tedder, T.F., 1992. Soluble L-selectin is present in human plasma at high levels and retains functional activity. J. Cell Biol. 119 229-238.

Sieling, P.A., Torrelles, J.B., Stenger, S., Chung, W., Burdick, A.E., Rea, T.H., et al., 2005. The human CD1-restricted T cell repertoire is limited to cross-reactive antigens: implications for host responses against immunologically related pathogens. J. Immunol. 174 2637-2644.

Silva, D., Ponte, C.G., Hacker, M.A., Antas, P.R., 2013. A whole blood assay as a simple, broad assessment of cytokines and chemokines to evaluate human immune responses to Mycobacterium tuberculosis antigens. Acta Trop. 127 75-81.

Skinner, M.A., Yuan, S., Prestidge, R., Chuk, D., Watson, J.D., Tan, P.L., 1997. Immunization with heat-killed Mycobacterium vaccae stimulates CD8+ cytotoxic T cells specific for macrophages infected with Mycobacterium tuberculosis. Infect. Immun. 65 4525-4530.

Smalley, D.M., Ley, K., 2005. L-selectin: mechanisms and physiological significance of ectodomain cleavage. J. Cell. Mol. Med. 9 255-266. 
Stanford, J., Stanford, C., Stansby, G., Bottasso, O., Bahr, G., Grange, J., 2009. The common mycobacterial antigens and their importance in the treatment of disease. Curr. Pharm. Des. 15 1248-1260.

Stanford, J.L., Stanford, C.A., O'Brien, M.E., Grange, J.M., 2008. Successful immunotherapy with Mycobacterium vaccae in the treatment of adenocarcinoma of the lung. Eur. J. Cancer 44 224-227.

Stebbing, J., Dalgleish, A., Gifford-Moore, A., Martin, A., Gleeson, C., Wilson, G., et al., 2012. An intra-patient placebo-controlled phase I trial to evaluate the safety and tolerability of intradermal IMM-101 in melanoma. Ann. Oncol. 23 1314-1319.

Thibodeau, J., Bourgeois-Daigneault, M.C., Lapointe, R., 2012. Targeting the MHC Class II antigen presentation pathway in cancer immunotherapy. Oncoimmunology 1908-916.

Truoc, L.V., Ly, H.M., Thuy, N.K., Trach, D.D., Stanford, C.A., Stanford, J.L., 2001. Vaccination against leprosy at Ben San Leprosy Centre, Ho Chi Minh City, Vietnam. Vaccine 19 3451-3458.

Tsuyuguchi, I., Kawasumi, H., Takashima, T., Tsuyuguchi, T., Kishimoto, S., 1990. Mycobacterium avium-Mycobacterium intracellular complex-induced suppression of T-cell proliferation in vitro by regulation of monocyte accessory cell activity. Infect. Immun. 58 13691378.

van Egmond, M., Bakema, J.E., 2013. Neutrophils as effector cells for antibody-based immunotherapy of cancer. Semin. Cancer Biol. 23 190-199.

von Reyn, C.F., Mtei, L., Arbeit, R.D., Waddell, R., Cole, B., Mackenzie, T., et al., 2010. Prevention of tuberculosis in Bacille Calmette-Guerin-primed, HIV-infected adults boosted with an inactivated whole-cell mycobacterial vaccine. AIDS 24 675-685.

Wang, Y., Kelly, C.G., Singh, M., McGowan, E.G., Carrara, A.S., Bergmeier, L.A., et al., 2002. Stimulation of Th1-polarizing cytokines, C-C chemokines, maturation of dendritic cells, and adjuvant function by the peptide binding fragment of heat shock protein 70. J. Immunol. 169 2422-2429.

Weir, R.E., Fine, P.E., Nazareth, B., Floyd, S., Black, G.F., King, E., et al., 2003. Interferongamma and skin test responses of schoolchildren in southeast England to purified protein derivatives from Mycobacterium tuberculosis and other species of mycobacteria. Clin. Exp. Immunol. 134 285-294.

Wingren, A.G., Parra, E., Varga, M., Kalland, T., Sjogren, H.O., Hedlund, G., et al., 1995. T cell activation pathways: B7, LFA-3, and ICAM-1 shape unique T cell profiles. Crit. Rev. Immunol. 15 235-253.

Wollenberg, A., Mommaas, M., Oppel, T., Schottdorf, E.M., Gunther, S., Moderer, M., 2002. Expression and function of the mannose receptor CD206 on epidermal dendritic cells in inflammatory skin diseases. J. Invest. Dermatol. 118 327-334. 
Yang, X.Y., Chen, Q.F., Li, Y.P., Wu, S.M., 2011. Mycobacterium vaccae as adjuvant therapy to anti-tuberculosis chemotherapy in never-treated tuberculosis patients: a meta-analysis. PLoS One 6 e23826.

Zipfel, P.F., Skerka, C., 2009. Complement regulators and inhibitory proteins. Nat. Rev. Immunol. 9 729-740.

Zuany-Amorim, C., Sawicka, E., Manlius, C., Le Moine, A., Brunet, L.R., Kemeny, D.M., et al., 2002. Suppression of airway eosinophilia by killed Mycobacterium vaccae-induced allergenspecific regulatory T-cells. Nat. Med. 8 625-629.

\section{Figures Captions}

Fig. 1. Whole blood stimulation with $M$. vaccae or $M$. obuense modulates surface expression of various receptors on monocytes. (A) Representative flow cytometry dot plots demonstrating the expression of CD62L, CD86 and CD184 on gated monocytes in whole blood that was left unstimulated or stimulated with $300 \mu \mathrm{g} / \mathrm{ml}$ of $M$. vaccae or $M$. obuense for $3 \mathrm{~h}$. Number within lower right quadrant indicates the percentage of receptor-positive monocytes out of the total monocyte population. (B) Representative flow cytometry histogram overlay plots showing the expression of CD11c, CD18 and HLA-DP,DQ,DR on gated monocytes in whole blood that was either left unstimulated or stimulated with $300 \mu \mathrm{g} / \mathrm{ml}$ of $M$. vaccae or $M$. obuense for $3 \mathrm{~h}$. Numbers in the plots correspond to the geometric mean fluorescence intensity (MFI) of receptor-positive monocytes in each condition (filled gray histogram: isotype control; black line histogram: unstimulated; red line histogram: M. vaccae; green line histogram: $M$. obuense).

Fig. 2. Toll-like receptor (TLR)-1 and/or TLR-2 blocking inhibit $M$. obuense-induced upregulation of CD11C and HLA-DR expression on monocytes in whole blood. Whole blood was preincubated with anti ( $\alpha$ )-TLR-1, $\alpha-T L R-2, \alpha-T L R-1 / 2, \alpha-T L R 6, \alpha-T L R-2 / 6, \alpha-T L R-4, \alpha-T L R-5$, isotype-matched control antibody IgA (for $\alpha$-TLR-2 and 5) or IgG (for $\alpha$-TLR-1, 4 and 6) for $1 \mathrm{~h}$ and then stimulated with (A) specific TLR2/1, TLR2, TLR4, TLR5, TLR2/6 ligands or (B) 300 $\mu \mathrm{g} / \mathrm{ml}$ of $M$. obuense (Mo) for $3 \mathrm{~h}$. The surface expression of CD11c and HLA-DR was analyzed on monocytes. Columns indicate the geometric mean fluorescence intensity (MFI) of CD11c or HLA-DR on monocytes. Data obtained are (A) representative of 1 out of 2 independent donors and (B) mean \pm SEM from 6 donors. Statistically significant differences were determined by paired t-test. ( ${ }^{*} p<0.05$ versus $M$. obuense). 
2 Fig. 3. Cytokine and chemokine levels in supernatants of whole blood cultures following 3 stimulation with $M$. vaccae or M. obuense. Scatter plots showing IL-6, IL-10, TNF- $\alpha$, CXCL8/IL-

4 8, MIP-1a, and RANTES levels in supernatants of whole blood cultures that were left 5 unstimulated or stimulated with $300 \mu \mathrm{g} / \mathrm{ml}$ of $M$. vaccae or M. obuense for $24 \mathrm{~h}$. Horizontal bars 6 indicate group mean values of cytokine or chemokine concentration of 15 donors. Statistically 7 significant differences were determined by Friedman test followed by Dunn's multiple 8 comparison post hoc test $\left({ }^{*} p<0.05\right.$ versus unstimulated).

Fig. 4. Induction of peripheral blood mononuclear cells (PBMCs) proliferative responses to $M$. vaccae and $M$. obuense. PBMCs were stimulated with different concentrations (1, 3, 10, 30, 100 and $300 \mu \mathrm{g} / \mathrm{ml}$ ) of $M$. vaccae or $M$. obuense for 7 days. Data are reported as (A) symbols with error bars showings mean of stimulation index \pm SEM from 4 donors or as (B) columns with error bars indicating mean of stimulation index \pm SEM from 12 donors. Statistically significant differences were determined either by Friedman test followed by Dunn's multiple comparison post hoc test $\left({ }^{*} p<0.05\right.$ versus 10 or $30 \mu \mathrm{g} / \mathrm{ml}$ of $M$. vaccae; ${ }^{\sigma} p<0.05$ versus $3 \mu \mathrm{g} / \mathrm{ml}$ of $M$. obuense) or by Wilcoxon signed rank test $\left({ }^{+} p<0.05\right.$ versus $M$. vaccae at the same concentration). 
1 Fig. 1

2

A
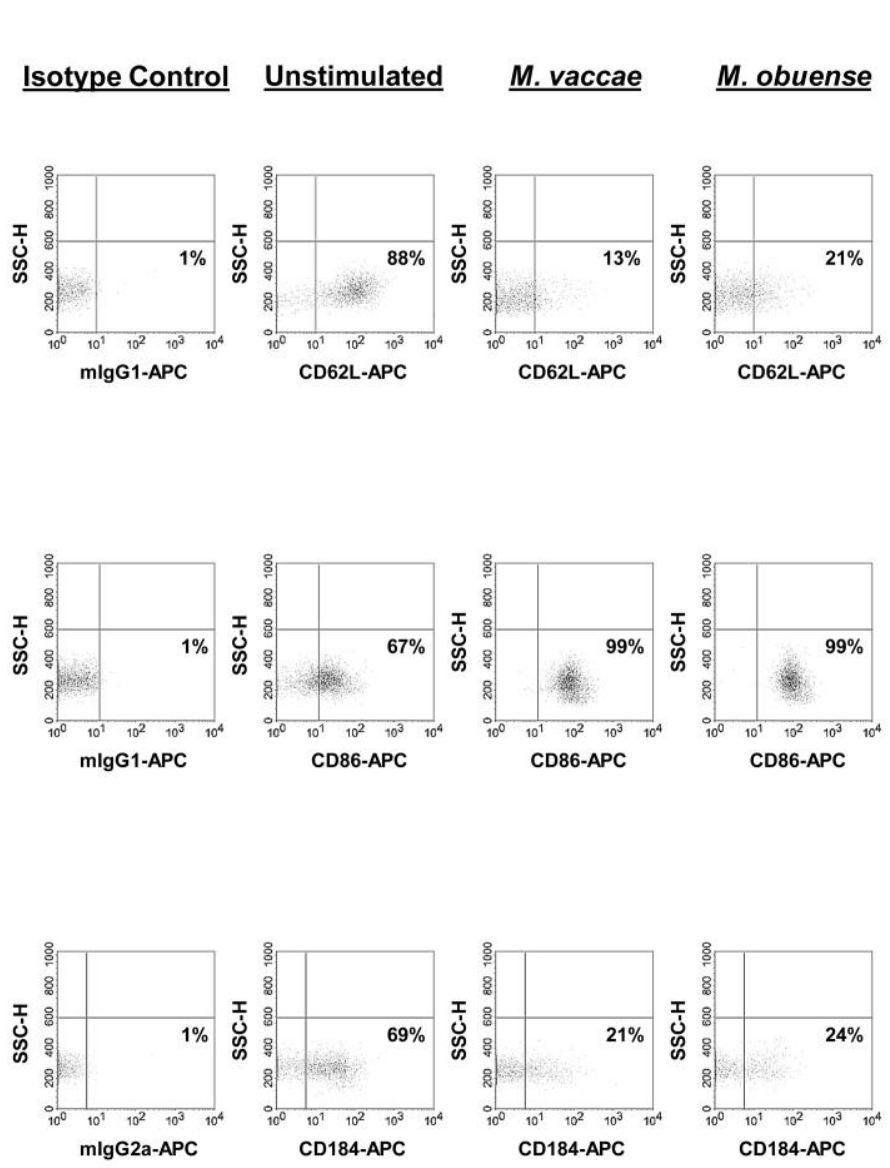

3

4

5

6

7

8

9

10

11

12

13

14
B
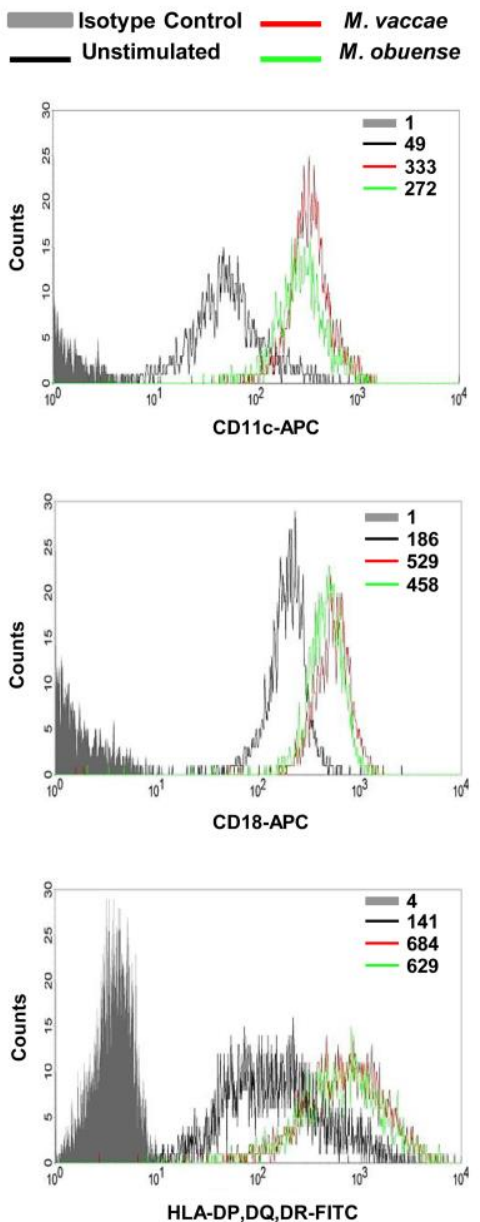
A
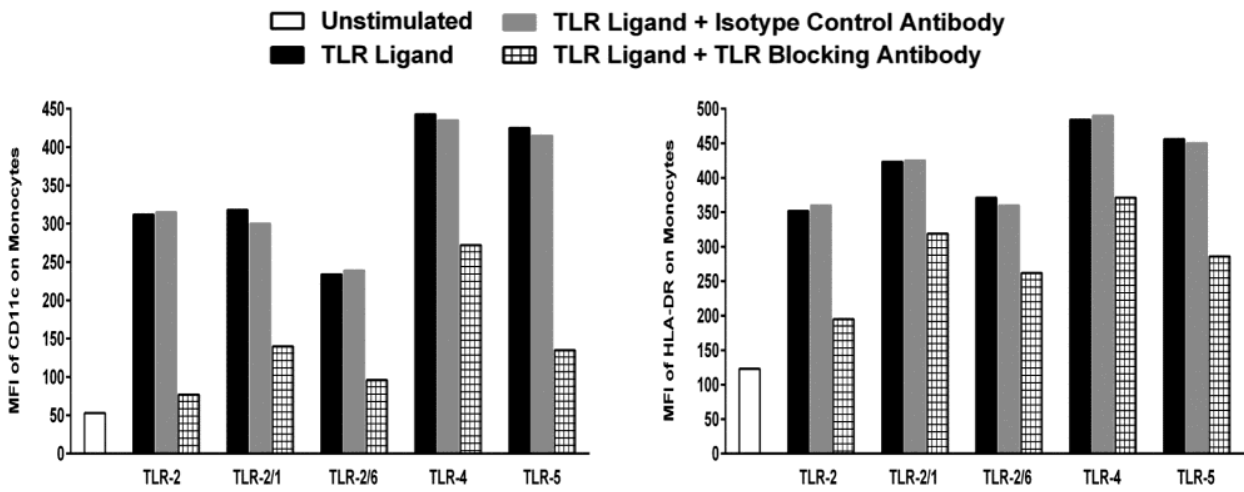

B
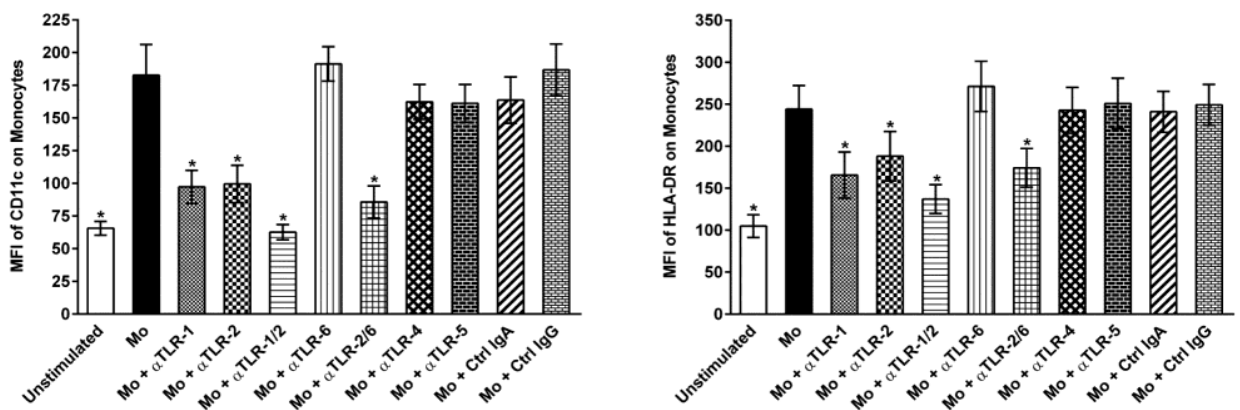
Fig. 3

Unstimulated X M. vaccae $\Delta$ M. obuense
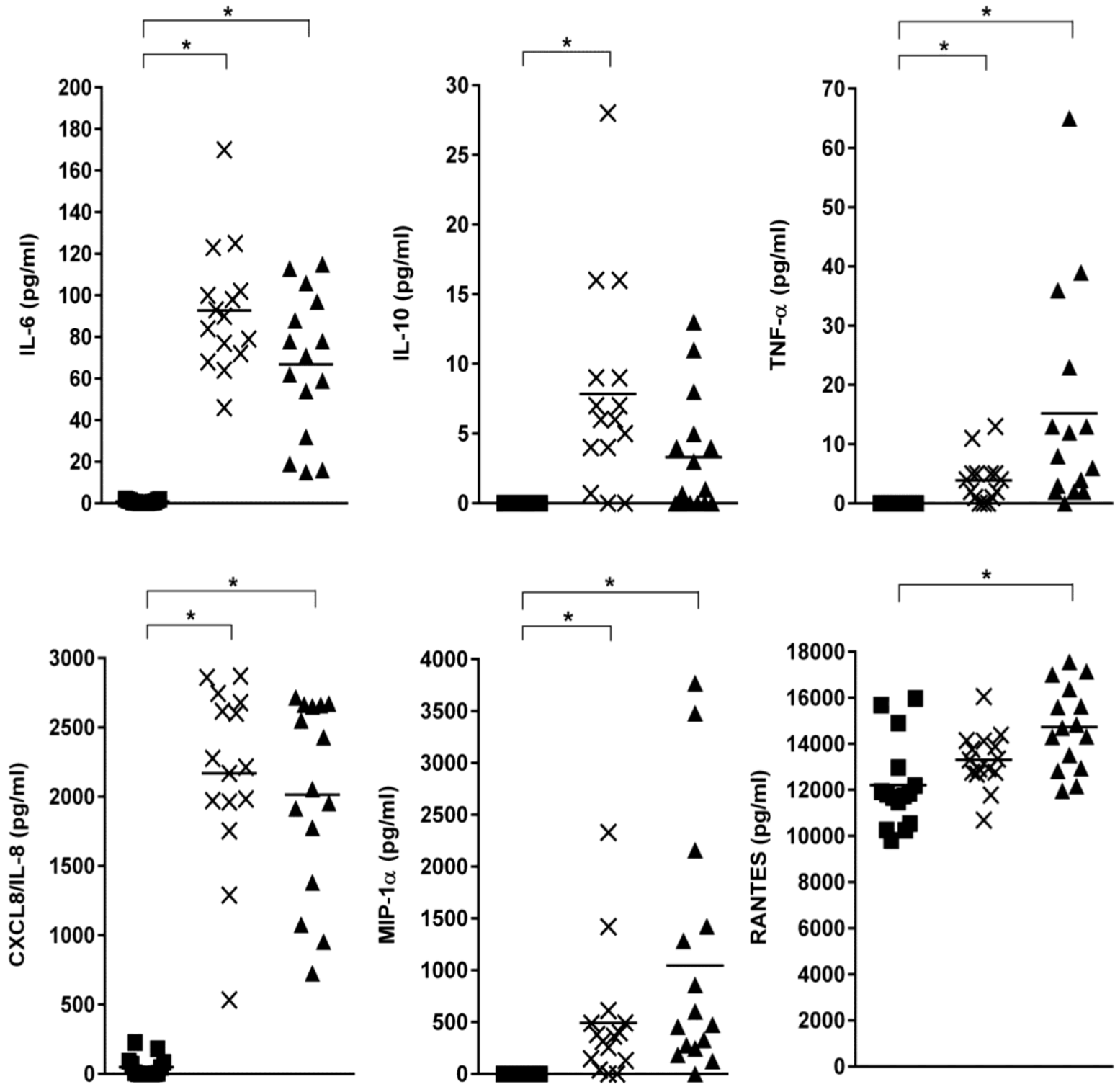
Fig. 4

A

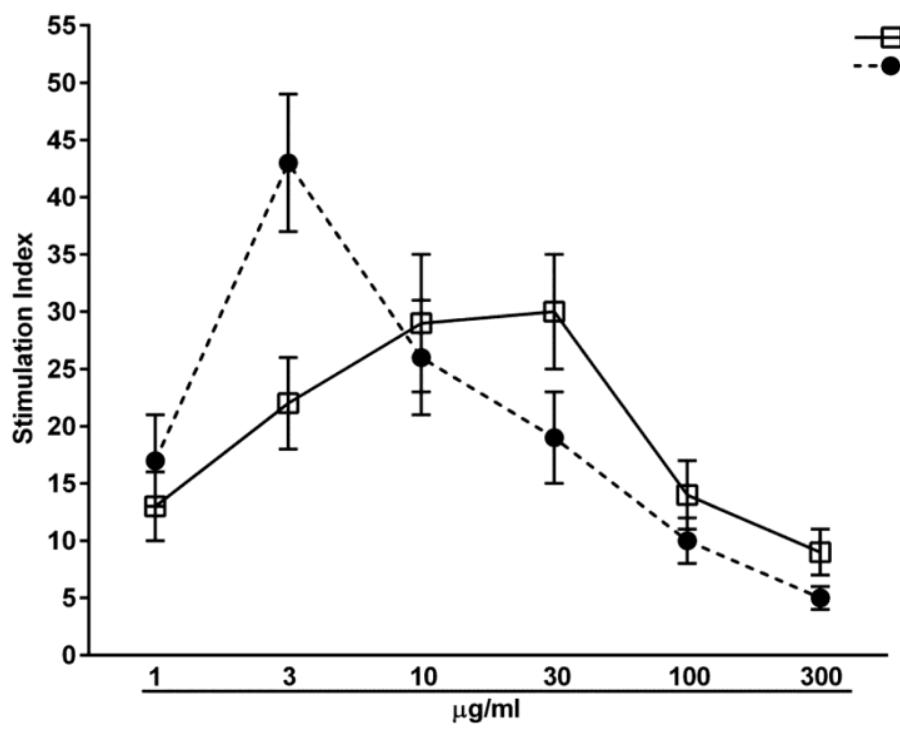

B

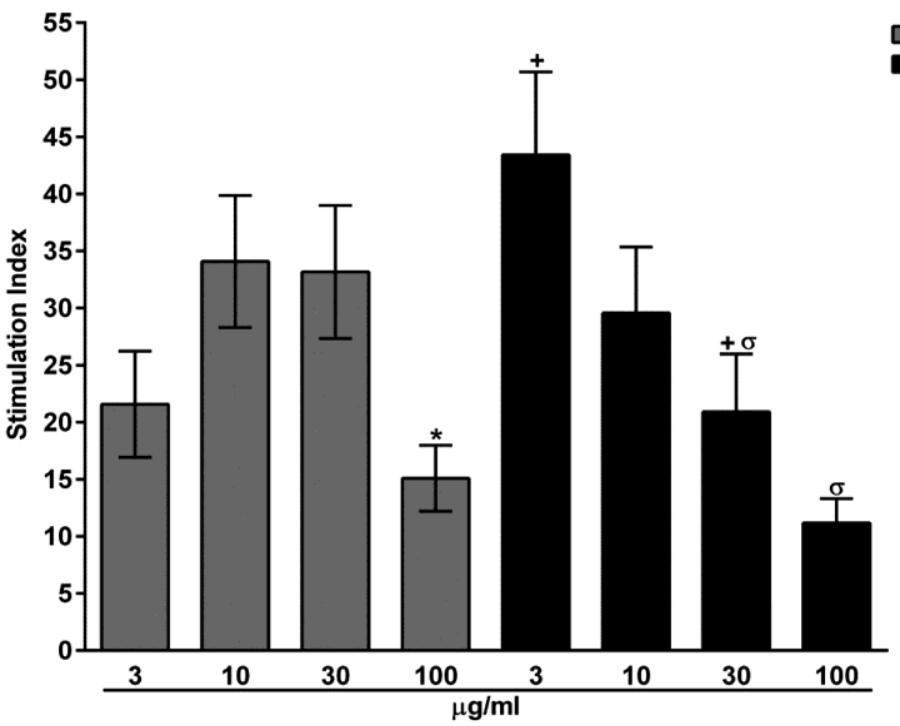

3

4

5

6 
Table 1.

2 Expression levels of surface receptors on monocytes in whole blood.

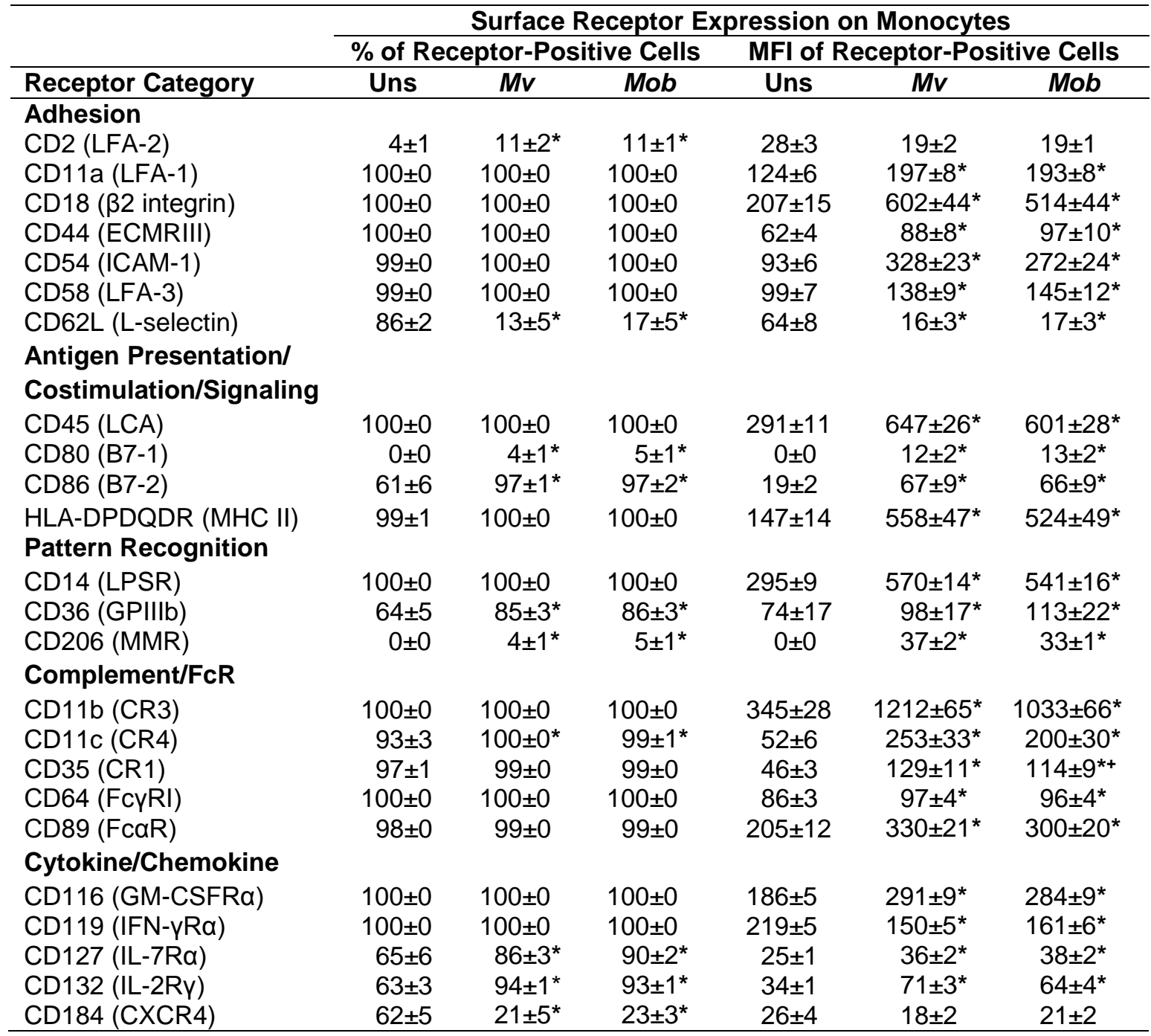


Table 2.

2 Expression levels of surface receptors on neutrophils in whole blood.

\begin{tabular}{|c|c|c|c|c|c|c|}
\hline \multirow{3}{*}{ Receptor Category } & \multicolumn{6}{|c|}{ Surface Receptor Expression on Neutrophils } \\
\hline & \multicolumn{3}{|c|}{$\%$ of Receptor-Positive Cells } & \multicolumn{3}{|c|}{ MFI of Receptor-Positive Cells } \\
\hline & Uns & $M v$ & Mob & Uns & $M v$ & Mob \\
\hline \multicolumn{7}{|l|}{ Adhesion } \\
\hline CD18 ( $\beta 2$ integrin) & $100 \pm 0$ & $100 \pm 0$ & $100 \pm 0$ & $144 \pm 7$ & $383 \pm 25^{*}$ & $251 \pm 16^{\star+}$ \\
\hline CD54 (ICAM-1) & $81 \pm 3$ & $98 \pm 1^{*}$ & $92 \pm 3^{+}$ & $27 \pm 3$ & $46 \pm 3$ & $36 \pm 2$ \\
\hline CD62L (L-selectin) & $100 \pm 0$ & $80 \pm 3^{*}$ & $94 \pm 1^{*}$ & $141 \pm 13$ & $53 \pm 5^{\star}$ & $105 \pm 9^{\star+}$ \\
\hline \multicolumn{7}{|c|}{$\begin{array}{l}\text { Antigen Presentation/ } \\
\text { Costimulation/Signaling }\end{array}$} \\
\hline CD45 (LCA) & $100 \pm 0$ & $100 \pm 0$ & $100 \pm 0$ & $87 \pm 4$ & $246 \pm 14^{*}$ & $144 \pm 8^{+}$ \\
\hline $\begin{array}{l}\text { HLA-A,B,C (MHC I) } \\
\text { Complement/Fc }\end{array}$ & $100 \pm 0$ & $99 \pm 0$ & $100 \pm 0$ & $104 \pm 14$ & $78 \pm 11^{*}$ & $100 \pm 14^{+}$ \\
\hline CD11b (CR3) & $100 \pm 0$ & $100 \pm 0$ & $100 \pm 0$ & $237 \pm 15$ & $1074 \pm 76^{*}$ & $563 \pm 46^{+}$ \\
\hline CD11c (CR4) & $89 \pm 3$ & $99 \pm 1 *$ & $95 \pm 3^{*}$ & $30 \pm 2$ & $78 \pm 7^{\star}$ & $51 \pm 5^{\star+}$ \\
\hline CD16 (FCyRIII) & $100 \pm 0$ & $99 \pm 0$ & $99 \pm 0$ & $1731 \pm 96$ & $2368 \pm 137^{*}$ & $2008 \pm 148^{*+}$ \\
\hline CD35 (CR1) & $95 \pm 1$ & $100 \pm 0^{*}$ & $99 \pm 1$ & $27 \pm 2$ & $114 \pm 9^{*}$ & $54 \pm 5^{\star+}$ \\
\hline CD64 (FcyRI) & $16 \pm 4$ & $24 \pm 5^{*}$ & $21 \pm 5^{*}$ & $17 \pm 2$ & $17 \pm 2$ & $18 \pm 2$ \\
\hline CD89 (FcaR) & $100 \pm 0$ & $100 \pm 0$ & $100 \pm 0$ & $182 \pm 16$ & $332 \pm 30^{*}$ & $215 \pm 17^{+}$ \\
\hline \multicolumn{7}{|c|}{ Cytokine/Chemokine } \\
\hline CD119 (IFN-yRa) & $100 \pm 0$ & $100 \pm 0$ & $100 \pm 0$ & $80 \pm 3$ & $61 \pm 2^{*}$ & $69 \pm 3^{*+}$ \\
\hline CD184 (CXCR4) & $82 \pm 2$ & $57 \pm 3^{*}$ & $71 \pm 3^{*+}$ & $30 \pm 2$ & $27 \pm 2$ & $29 \pm 1$ \\
\hline
\end{tabular}

The table comprises all the CD antigens that showed statistically significant variations of

4 expression on neutrophils in whole blood that was left unstimulated (Uns) or stimulated with

$5300 \mu \mathrm{g} / \mathrm{ml}$ of $M$. vaccae $(M v)$ or $M$. obuense $(M o b)$ for $3 \mathrm{~h}$. Data represent mean \pm SEM of the

6 percentage (\%) and geometric mean fluorescence intensity (MFI) of receptor-positive

7 neutrophils of 15 donors. Statistically significant differences were determined by Friedman test

8 followed by Dunn's multiple comparison post hoc test $\left({ }^{*} p<0.05\right.$ versus Uns; ${ }^{+} p<0.05$ versus $9 \mathrm{Mv})$. 
A

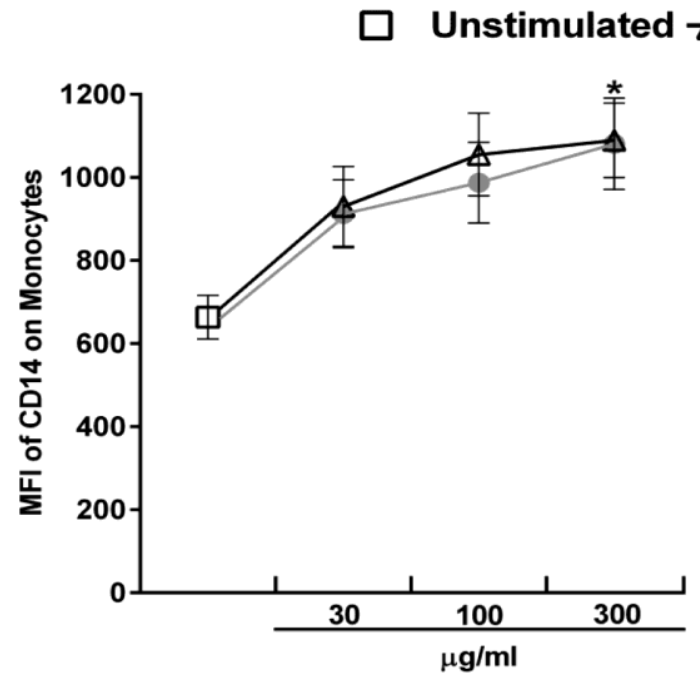

M. vaccae

M. obuense

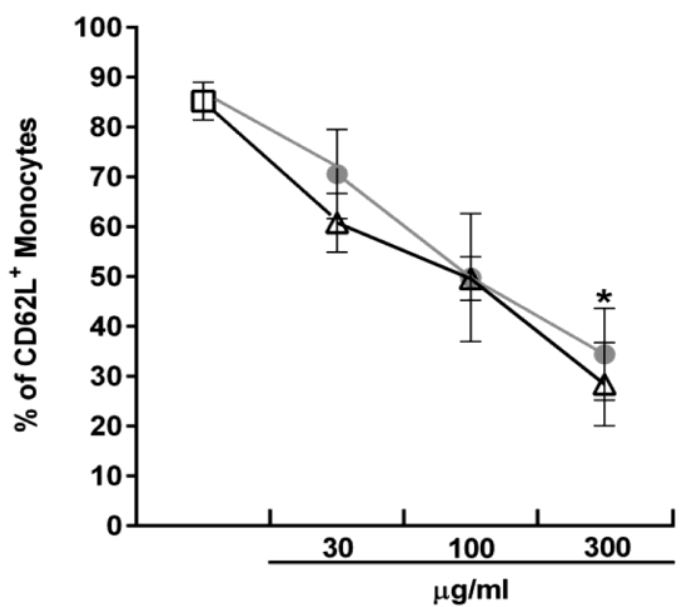

B

M. vaccae

M. obuense
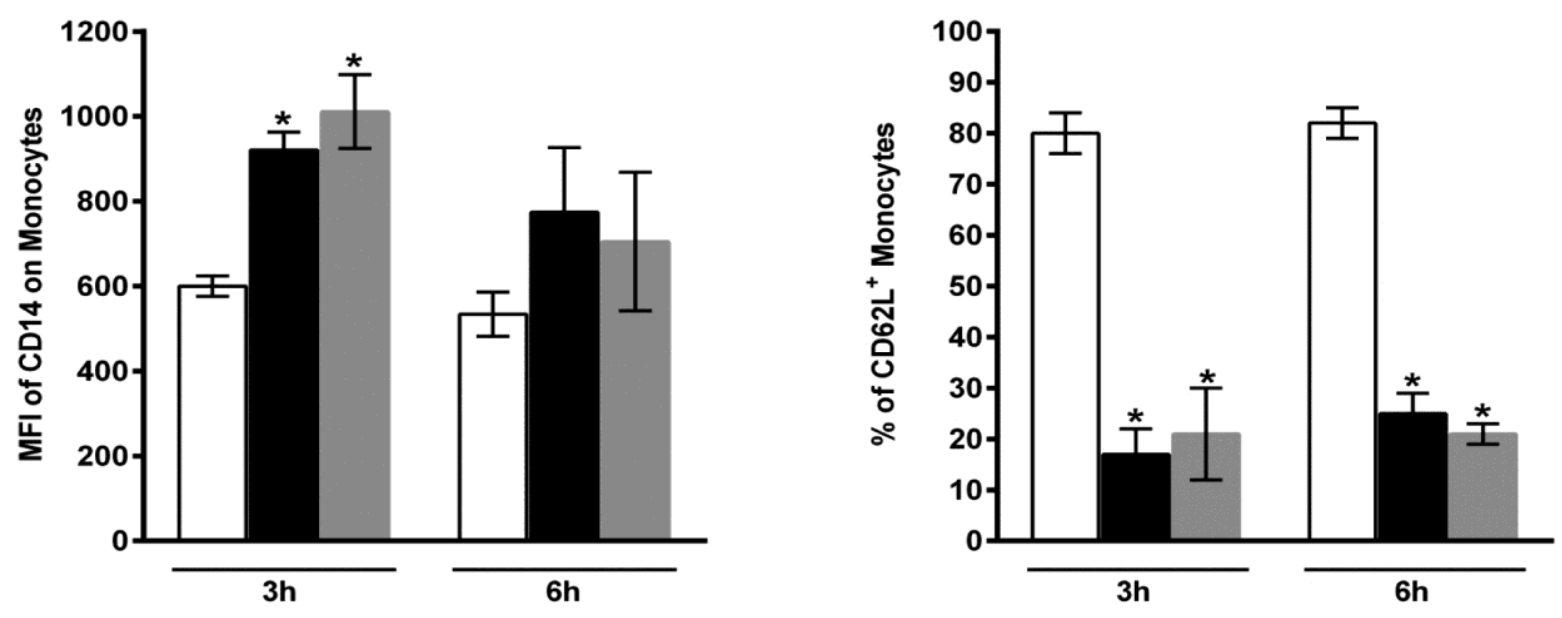

Supplementary Figure 1. Optimization of whole blood stimulation conditions with $M$. vaccae or $M$. obuense. (A) Dose-response evaluation for the effect of whole blood stimulation with different concentrations of $M$. vaccae or $M$. obuense $(30,100$ and $300 \mu \mathrm{g} / \mathrm{ml})$ for $3 \mathrm{~h}$ on the expression levels of CD14 and CD62L presented as geometric mean fluorescence intensity (MFI) and percentage of positive monocytes, respectively. Symbols and error bars indicate mean values and SEM ( $n=5)$. (B) Time-response evaluation for the effect of whole blood stimulation with $300 \mu \mathrm{g} / \mathrm{ml}$ of $M$. vaccae or $M$. obuense for 3 or $6 \mathrm{~h}$ on the expression levels of CD14 and CD62L presented as geometric MFI and percentage of positive monocytes, respectively. Columns and error bars indicate mean values and SEM $(n=3)$.

${ }^{*} p<0.05$ versus unstimulated. 
$\square$ Unstimulated $\square$ M. vaccae $\square$ M. obuense

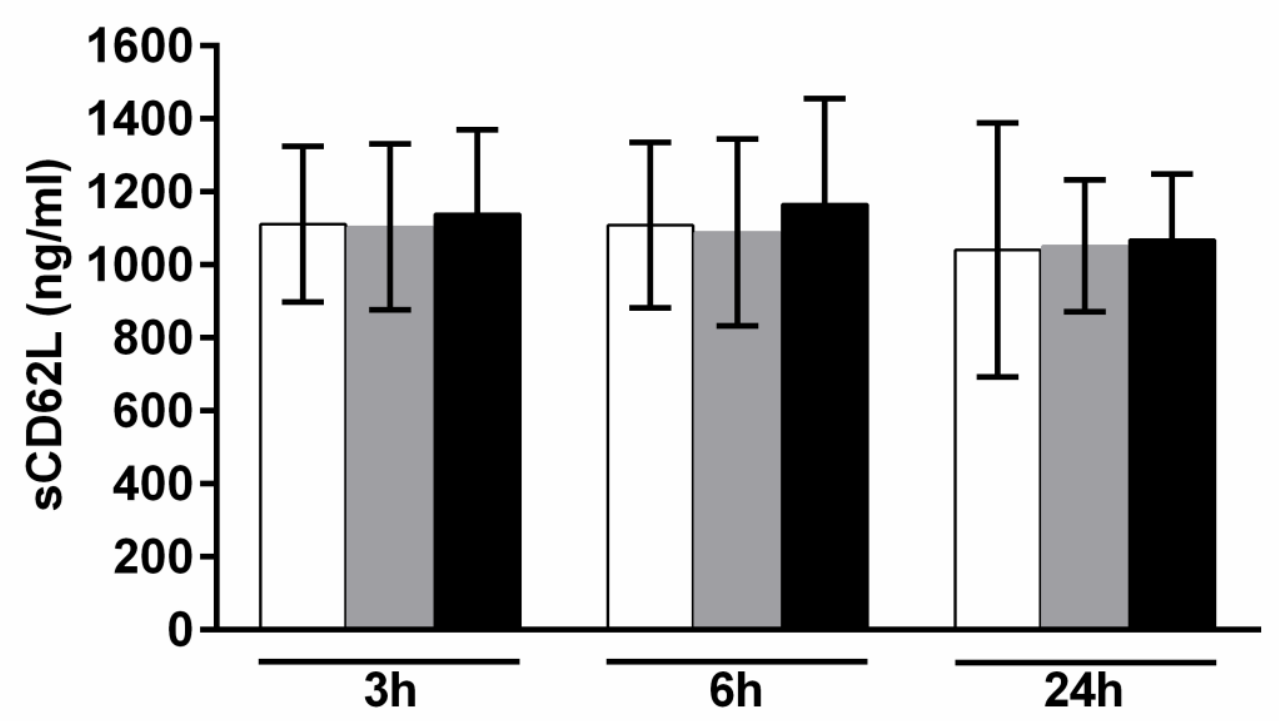

Supplementary Figure 2. Soluble (s) CD62L levels in supernatants of whole blood cultures. sCD62L levels in supernatants of whole blood cultures that were left unstimulated or stimulated with $300 \mu \mathrm{g} / \mathrm{ml}$ of $M$. vaccae or $M$. obuense for 3,6 , or $24 \mathrm{~h}$. Columns indicate the mean sCD62L levels of 4 donors. Error bars represent SEM. 
Supplementary Table 1.

2 Surface receptors with unchanged expression levels on monocytes in whole blood.

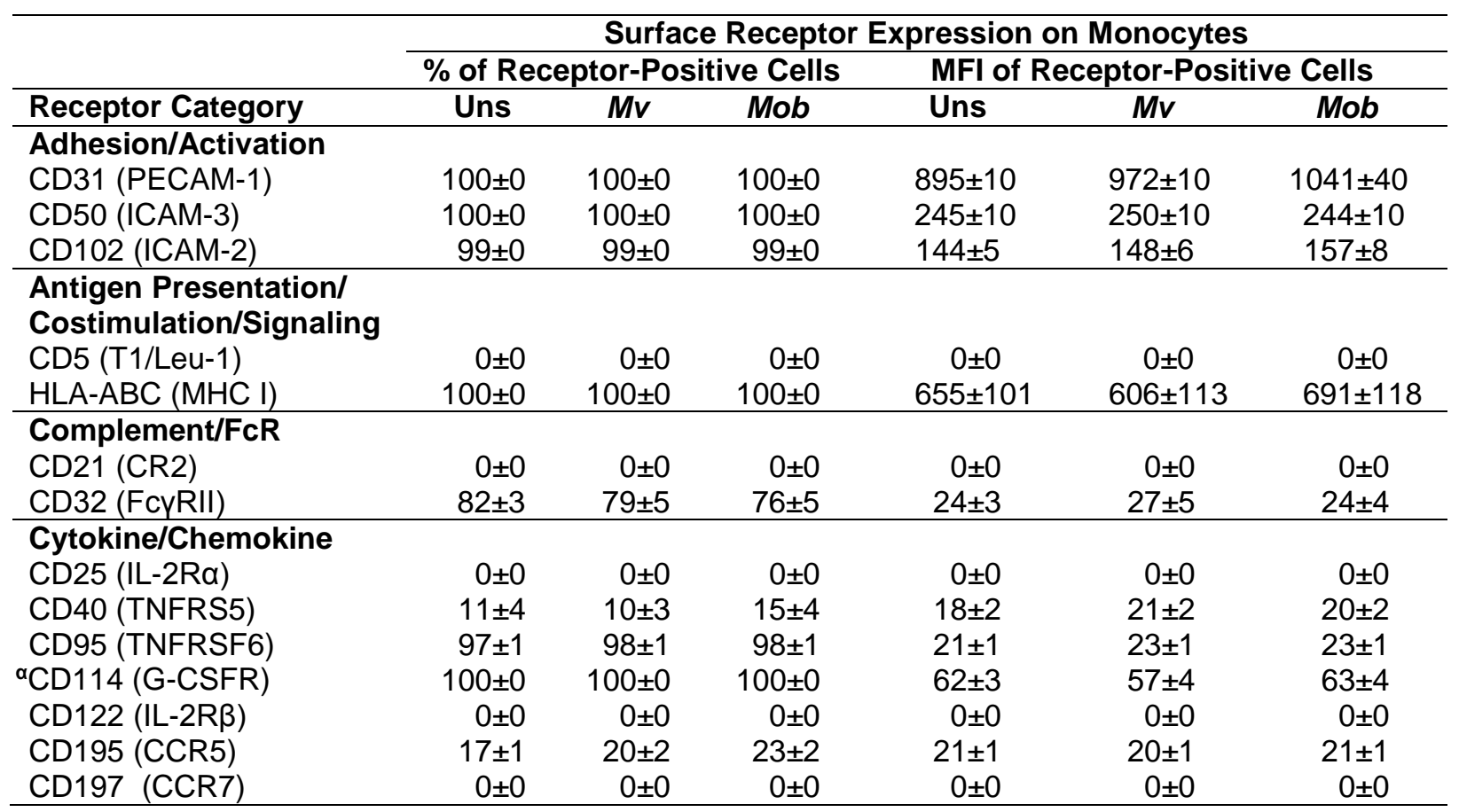

The table comprises all the receptors which did not show any significant variation of expression on monocytes in whole blood that was left unstimulated (Uns) or stimulated with $300 \mu \mathrm{g} / \mathrm{ml} \mathrm{of} M$. vaccae $(M v)$ or $M$. obuense $(M o b)$ for $3 \mathrm{~h}$. Data represent mean \pm SEM of the percentage (\%) and geometric mean fluorescence intensity (MFI) of receptor-positive monocytes of 15 donors. aExperiments conducted on 9 donors. 


\section{Supplementary Table 2.}

Surface receptors with unchanged expression levels on neutrophils in whole blood.

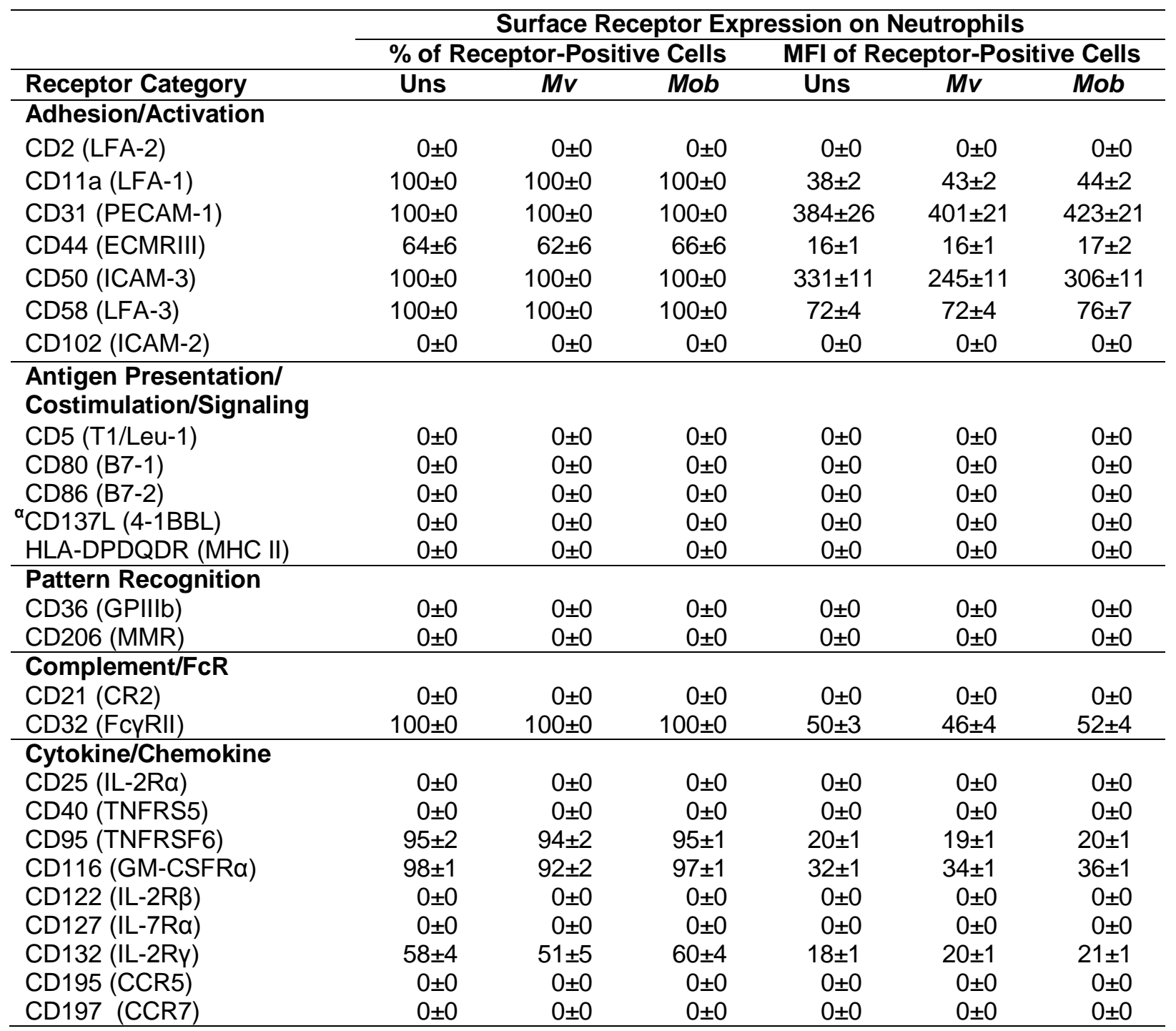

The table comprises all the receptors which did not show any significant variation of expression on neutrophils in whole blood that was left unstimulated (Uns) or stimulated with $300 \mu \mathrm{g} / \mathrm{ml}$ of $M$. vaccae $(M v)$ or $M$. obuense $(M o b)$ for $3 \mathrm{~h}$. Data represent mean \pm SEM of the percentage (\%) and geometric mean fluorescence intensity (MFI) of receptor-positive neutrophils of 15 donors. aExperiments conducted on 9 donors. 